\title{
Activity-Induced Synaptic Structural Modifications by an Activator of Integrin Signaling at the Drosophila Neuromuscular Junction
}

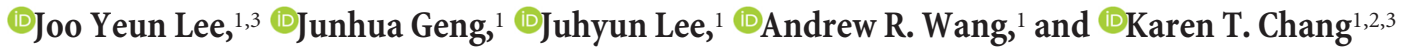 \\ 'Zilkha Neurogenetic Institute and ${ }^{2}$ Department of Cell and Neurobiology, Keck School of Medicine, and ${ }^{3}$ Neuroscience Graduate Program, University of \\ Southern California, Los Angeles, California 90089
}

Activity-induced synaptic structural modification is crucial for neural development and synaptic plasticity, but the molecular players involved in this process are not well defined. Here, we report that a protein named Shriveled (Shv) regulates synaptic growth and activity-dependent synaptic remodeling at the Drosophila neuromuscular junction. Depletion of Shv causes synaptic overgrowth and an accumulation of immature boutons. We find that Shv physically and genetically interacts with $\beta$ PS integrin. Furthermore, Shv is secreted during intense, but not mild, neuronal activity to acutely activate integrin signaling, induce synaptic bouton enlargement, and increase postsynaptic glutamate receptor abundance. Consequently, loss of Shv prevents activity-induced synapse maturation and abolishes post-tetanic potentiation, a form of synaptic plasticity. Our data identify Shv as a novel trans-synaptic signal secreted upon intense neuronal activity to promote synapse remodeling through integrin receptor signaling.

Key words: activity-dependent structural remodeling; Drosophila; integrin; neuromuscular junction; Shriveled; synaptic boutons

\section{Significance Statement}

The ability of neurons to rapidly modify synaptic structure in response to neuronal activity, a process called activity-induced structural remodeling, is crucial for neuronal development and complex brain functions. The molecular players that are important for this fundamental biological process are not well understood. Here we show that the Shriveled (Shv) protein is required during development to maintain normal synaptic growth. We further demonstrate that Shv is selectively released during intense neuronal activity, but not mild neuronal activity, to acutely activate integrin signaling and trigger structural modifications at the Drosophila neuromuscular junction. This work identifies Shv as a key modulator of activity-induced structural remodeling and suggests that neurons use distinct molecular cues to differentially modulate synaptic growth and remodeling to meet synaptic demand.

\section{Introduction}

The synapse is a highly dynamic structure that undergoes rapid changes in morphology and strength in response to synaptic activity (Berninger and Bi, 2002; Fiala et al., 2002; Chklovskii et al., 2004; Bourne and Harris, 2008; Holtmaat and Svoboda, 2009). Such activity-induced structural and functional plasticity under-

\footnotetext{
Received Oct. 7, 2016; revised Feb. 8, 2017; accepted Feb. 14, 2017.

Author contributions: J.Y.L. and K.T.C. designed research; J.Y.L., J.G., J.L., and A.R.W. performed research; J.Y.L. and K.T.C. analyzed data; J.Y.L. and K.T.C. wrote the paper.

K.T.C. is supported by grants from the National Institutes of Health (NS-080946), the Alzheimer's Association, and Global Down Syndrome Foundation. We thank Drs. R. Xi (National Institute of Biological Science, Beijing, People's Republic of China), A. DiAntonio (Washington University at St. Louis, St. Louis, M0) for prompt and generous sharing of fly stocks and antibodies. We also thank the Developmental Hybridoma Bank of the University of lowa (lowa City, IA) for multiple antibodies. In addition, we thank the members of the Chang laboratory for discussions.

Correspondence should be addressed to Karen T. Chang, Zilkha Neurogenetic Institute, University of Southern California, Los Angeles, CA 90089. E-mail: changkt@usc.edu.

DOI:10.1523/JNEUROSCI.3128-16.2017

Copyright $\odot 2017$ the authors $\quad 0270-6474 / 17 / 373246-18 \$ 15.00 / 0$
}

lies complex brain functions such as learning and memory and is associated with neurodevelopmental and psychiatric disorders (Fiala et al., 2002; Trachtenberg et al., 2002; Lamprecht and LeDoux, 2004; Xie et al., 2007). Multiple synaptic machineries that govern gene transcription and post-translational modification of synaptic molecules have been shown to play an important role in activity-induced synaptic plasticity by coordinating efficient synaptic transmission between presynaptic and postsynaptic compartments (Berninger and Bi, 2002; Lamprecht and LeDoux, 2004; Je et al., 2006; Cohen and Greenberg, 2008; Kim et al., 2015). However, despite a wealth of discoveries on molecules that play major roles in functional plasticity, mechanisms underlying activity-induced structural modifications are not well understood.

The synaptically located extracellular matrix (ECM) environment and cell adhesion molecules (CAMs) are thought to coordinate communication across the synaptic cleft to regulate 
synaptic growth, remodeling, and stabilization (Dityatev and Schachner, 2003; Yamagata et al., 2003; Rushton et al., 2009; Arikkath, 2010; Dityatev et al., 2010; Broadie et al., 2011). Among CAMs, integrin receptors are heterodimeric transmembrane receptors that enable intercellular signaling by bridging the ECM to the intracellular actin cytoskeleton (Campbell and Humphries, 2011). Emerging data suggest that integrin receptors are important for coordinating activity-dependent structural and functional modifications in Drosophila and mammalian nervous systems (Beumer et al., 1999, 2002; Rohrbough et al., 2000; Chavis and Westbrook, 2001; McGeachie et al., 2011; Dani et al., 2014). Functional blockade of integrin-ECM ligand interaction has been shown to significantly reduce consolidation of LTP in mammalian hippocampal neurons (Grooms and Jones, 1997; Stäubli et al., 1998; Chun et al., 2001; Chan et al., 2003, 2006; Huang et al., 2006) and activity-dependent synaptic plasticity in Drosophila (Rohrbough et al., 2000; Dani et al., 2014).

While evidence suggests that integrin activation is essential for synaptic plasticity (Bahr et al., 1997; Beumer et al., 1999; Rohrbough et al., 2000; Chavis and Westbrook, 2001; Chun et al., 2001; Kramár et al., 2006; McGeachie et al., 2011; Dani et al., 2014), very little is known about the molecular cues that contribute to integrin activation during activity-induced synaptic plasticity. The Drosophila glutamatergic neuromuscular junction (NMJ) is an excellent model to identify molecular players modulating activity-induced structural remodeling due to its powerful genetics and well conserved signaling pathways. There are five $\alpha$ integrin subunits and two $\beta$ integrin subunits ( $\beta P S$ and $\beta \nu$ ) in Drosophila (Brown, 1993; Gotwals et al., 1994). Several integrin ligands, such as Tiggrin (Fogerty et al., 1994), laminin A (Inoue and Hayashi, 2007), Teneurin (Graner et al., 1998; Mosca, 2015), have been reported. However, to date, laminin is the only known integrin ligand with its release regulated in an activity-dependent manner at the Drosophila NMJ (Tsai et al., 2012a). Synaptic activity has been shown to downregulate laminin release from the postsynaptic muscle cells, thereby permitting synaptic growth and expansion through reduced integrin activation (Tsai et al., 2012a). Here, we present evidence that an activator of integrin signaling, Shriveled (Shv; Lee et al., 2016), is released presynaptically in an activity-dependent manner at the Drosophila NMJ. We demonstrate that Shv regulates proper synaptic growth at the Drosophila NMJ and plays an important role in activity-induced structural modifications via the integrin signaling pathway. We find that Shv is acutely secreted during intense neuronal activity, but not during mild activity, to promote synaptic bouton enlargement and increase postsynaptic glutamate receptor abundance. We further demonstrate that Shv release is required for synaptic plasticity. Together, our data suggest that Shv is a novel trans-synaptic protein secreted by neurons in an activity-dependent manner to activate integrin signaling and induce synaptic structural modifications at the Drosophila NMJ.

\section{Materials and Methods}

Drosophila genetics. Flies were cultured at $25^{\circ} \mathrm{C}$ on standard cornmeal, yeast, sugar, and agar medium under a $12 \mathrm{~h}$ light/dark cycle unless noted otherwise. To prevent the lethality caused by the silencing of integrin in muscles, the tubulin-GAL80 ${ }^{t s}$ line was used to control GAL4 expression temporally. Vials were kept at $18^{\circ} \mathrm{C}$ to block GAL4 activity during embryonic development and shifted to the $29^{\circ} \mathrm{C}$ after L1 stage to activate GAL4 expression. White-eyed flies $\left(w^{1118}\right)$ were used as wild type throughout all experiments. The following fly lines were used [Bloomington Drosophila Stock Center at Indiana University (Bloomington, IN) stock number is in parentheses]: $m y s^{t s I}(\# 3169), 24 B-G A L 4$ (\#1767), tubulin-GAL80 $0^{\text {ts }}$ (\#7016), UAS-mys (a gift from from R. Xi, National
Institute of Biological Science, Beijing, People's Republic of China), $n S y b-G A L 4$ (a gift from Dr. Julie Simpson, University of California, Santa Barbara, CA; Pauli et al., 2008), and $s h v^{1}$ (Lee et al., 2016). UAS-shv and $U A S-N o S P-s h v$ were cloned into pINDY6 vector containing the HA tag as described previously (Lee et al., 2016). Transgenic RNAi flies UAS-shvRNAi (stock \#108576), UAS-mys-RNAi (stock \#29619), and UAS$\beta_{\nu}$ Integrin-RNAi (stock \#40895) were obtained from Vienna Drosophila Resource Center (Vienna, Austria). UAS-shv-GFP and UAS-NoSP-shvGFP flies were generated by inserting EGFP sequence to the $\mathrm{C}$ terminus of full-length Shv and Shv with deleted signal peptide and subcloned into the pINDY6 vector, respectively. Transgenic files were generated by standard transformation method. All other stocks and standard balancers were obtained from the Bloomington Drosophila Stock Center at Indiana University. Flies of both sexes were used, except for experiments involving expression of transgenes in $m y s^{t s l}$ background, in which only males were used ( $m y s^{t s l}$ located on the X chromosome).

Immunocytochemistry. Third-instar larvae were dissected in the following $\mathrm{Ca}^{2+}$ free dissection buffer: $\mathrm{NaCl} 128 \mathrm{~mm}, \mathrm{KCl} 2 \mathrm{~mm}, \mathrm{MgCl}_{2} 4.1$ $\mathrm{mm}$, sucrose $35.5 \mathrm{~mm}$, HEPES $5 \mathrm{~mm}$, and EGTA $1 \mathrm{~mm}$. Motor nerves were cut, and dissected preparations were fixed in $4 \%$ paraformaldehyde solution for $25 \mathrm{~min}$ at room temperature (except Bouin's fixative was used for GluR staining). Fixed samples were then washed with $0.1 \%$ Triton X-100 in PBS (PBST) or with PBS for detergent-free condition. Samples were blocked with 5\% normal goat serum in PBST or PBS, as indicated. Primary antibodies were diluted in blocking solution and used as follows: rabbit anti-Shv, 1:400 (Lee et al., 2016); mouse anti-Dlg (Discs large), 1:500 [4F3, Developmental Studies Hybridoma Bank at the University of Iowa (DSHB), Iowa City, IA]); rat anti-Elav, 1:500 (7E8A10); mouse anti-Repo, 1:10 (8D12, DSHB); rabbit anti-phosphoFAK, 1:50 (Abcam); mouse anti-NC82, 1:20 (DSHB); rabbit anti-GluRIII, 1:1000 (from Dr. A. DiAntonio, Washington University at St. Louis, St. Louis, MO); GluRIIA, 1:10 (8B4D2, DSHB) GluRIIB, 1:2500 (from Dr. A. DiAntonio); and anti-GFP, 1:1000 (Acris). Cy3-conjugated anti-HRP, 1:100 (Jackson ImmunoResearch). A $100 \mu \mathrm{M}$ working stock of Actin-stain 488 fluorescent phalloidin (Cytoskeleton) was used to stain postsynaptic muscle. Secondary antibodies used were Alexa Fluor 488 or 405 conjugated, at a dilution of 1:250 (Invitrogen).

Synaptic activity stimulation. Third-instar larval body walls were dissected in normal HL-3 solution without $\mathrm{Ca}^{2+}(\mathrm{NaCl} 110 \mathrm{~mm}, \mathrm{KCl} 5 \mathrm{~mm}$, $\mathrm{MgCl}_{2} 10 \mathrm{~mm}$, sucrose $30 \mathrm{~mm}$, HEPES $5 \mathrm{~mm}$, EGTA $1 \mathrm{~mm}$, trehalose $5 \mathrm{~mm}$, and $\mathrm{NaO}_{3} 10 \mathrm{~mm}, \mathrm{pH}$ 7.2), leaving the brain and peripheral nerves intact. High $\mathrm{K}^{+}$stimulation was achieved by applying a solution with $90 \mathrm{~mm}$ $\mathrm{KCl}$ in HL-3 solution with $\mathrm{Ca}^{2+}\left(\mathrm{NaCl} 25 \mathrm{~mm}, \mathrm{KCl} 90 \mathrm{~mm}, \mathrm{MgCl}_{2} 10 \mathrm{~mm}\right.$, $\mathrm{CaCl}_{2} 1.5 \mathrm{~mm}$, sucrose $30 \mathrm{~mm}$, HEPES $5 \mathrm{~mm}$, trehalose $5 \mathrm{~mm}$, and $\mathrm{NaHCO}_{3} 10 \mathrm{~mm}, \mathrm{pH} 7.2$ ) for $10 \mathrm{~min}$ and replaced with a resting solution (normal HL-3 solution without $\mathrm{Ca}^{2+}$ ) for $2 \mathrm{~min}$ followed by $25 \mathrm{~min}$ fixation. For the experiment with exogenous Shv application, dissected preps were incubated with $1 \mu \mathrm{g} / \mathrm{ml}$ purified Shv-HA or Shv ${ }^{\mathrm{LNV}}$-HA in HL-3 solution for $10 \mathrm{~min}$. The $3 \times$ spaced high $\mathrm{K}^{+}$depolarization paradigm was adapted from Vasin et al. (2014) except the CNS and nerves were kept intact in our preparation. The $5 \times$ spaced high $\mathrm{KCl}$ stimulation paradigm was adapted from Ataman et al. (2008). For inhibition of transcription or translation, dissected NMJs were preincubated with either 5 $\mu \mathrm{M}$ actinomycin-D (Biotium) or $200 \mu \mathrm{M}$ cycloheximide (Amresco) for $20 \mathrm{~min}$ in HL-3 solution, and the inhibitor was also included in normal/ high $\mathrm{K}^{+}$solutions and applied throughout stimulation protocol (Frank et al., 2006; Koon et al., 2011). For electrical stimulation, the severed nerve was stimulated by suction electrode at either 1 or $10 \mathrm{~Hz}$ for $5 \mathrm{~min}$ in normal HL-3 solution.

Image quantification. Images of the synaptic terminal from NMJ 6/7 in A2 and A3 were captured using a Zeiss LSM5 Confocal Microscope using a $63 \times 1.6$ numerical aperture oil-immersion objective with a $0.7 \times$ or $1 \times$ zoom. The average bouton size was determined by outlining individual Type $1 \mathrm{~b}$ bouton using ImageJ. The number of boutons were calculated by counting HRP-labeled boutons, then they were normalized to the surface of muscle areas $6 / 7$ taken with a $10 \times$ objective. There was no significant difference between the surface muscle areas of different genotypes examined. Synaptic boutons without Dlg, NC82, or GluRIII staining were counted as a ghost boutons (Ataman et al., 2008; Vasin et al., 2014). The 
ghost bouton index was calculated by counting the number of ghost boutons per NMJ and normalizing it to the total number of synaptic boutons in the same synapse. The relative fold change in ghost bouton index was determined by normalizing the value to control. Since new boutons are thought to be formed from existing boutons (Menon et al., 2013), normalizing the ghost bouton number to the total bouton number per NMJ minimizes the bias caused by differences in the abundance of existing boutons. To analyze Shv levels at the NMJ stained with Shv antibody and Cy3-HRP under detergent-free condition, we first outlined an individual synaptic bouton at a size that is $1.2 \mu \mathrm{m}$ larger in radius than the size of the bouton detected by HRP, and then measured the Shv signal in the selected area. As Shv is a secreted protein that is present extracellularly, we selected an area larger than the synaptic bouton area to determine Shv level at the synapse. A bouton size of $1.2 \mu \mathrm{m}$ is selected since it is approximately twice the thickness of Dlg staining (postsynaptic marker), which on average extends $0.63 \pm 0.01 \mu \mathrm{m}$ in radius beyond HRP staining $(n=8 \mathrm{NMJs})$. Please note that Dlg colabeling was not possible under the detergent free condition. When comparing intensity across genotypes, the exposure time was kept constant for all genotypes per experiment. Staining intensities were measured by normalizing the fluorescence intensity to bouton area outlined by either HRP or Discslarge using ImageJ. All values were normalized to control within the same experimental set.

Electrophysiology. Third-instar larvae were dissected and then bathed in a modified HL-3 solution $\left(\mathrm{NaCl} 70 \mathrm{~mm}, \mathrm{KCl} 5 \mathrm{~mm}, \mathrm{MgCl}_{2} 10 \mathrm{~mm}\right.$, sucrose $115 \mathrm{~mm}$, HEPES $5 \mathrm{~mm}$, trehalose $5 \mathrm{~mm}$, and $\mathrm{NaHCO}_{3} 10 \mathrm{~mm}, \mathrm{pH}$ 7.1) with 0.2 or $0.5 \mathrm{~mm} \mathrm{CaCl}_{2}$ as indicated. Current-clamp recordings were performed on muscle area 6 in abdominal segments A2 or A3, and severed ventral nerves were stimulated with suction electrodes at $0.3 \mathrm{~ms}$ stimulus duration. The recording electrode with resistance between 15 and $40 \mathrm{M} \Omega$ was filled with $3 \mathrm{M} \mathrm{KCl}$, and data with resting potential more hyperpolarized than $-60 \mathrm{mV}$ were analyzed. Datasets were rejected if resting potentials deviated by $>10 \%$ during the recording and if EPSP amplitude dropped abruptly, indicating that stimulated nerves did not fully function throughout the recording. Data were acquired using an Axopatch 200B Amplifier, digitized using a Digidata 1440A, and controlled using pClamp 10.3 software (Molecular Devices). Data were analyzed using MiniAnalysis (Synaptosoft), Clampfit (Molecular Devices), and Microsoft Excel. The average EPSP was corrected using nonlinear summation.

Western blotting. To detect Shv levels in flies, protein extracts were obtained by homogenizing flies in RIPA lysis buffer $(50 \mathrm{~mm}$ Tris- $\mathrm{HCl}$, pH7.5, 1\% NP-40, 0.5\% NaDoc, 150 mм NaCl, 0.1\% SDS, 2 mм EDTA, $50 \mathrm{~mm} \mathrm{NaF}, 1 \mathrm{~mm} \mathrm{Na}_{3} \mathrm{VO}_{4}, 250 \mathrm{~nm}$ cyclosporin A, and protease inhibitor cocktail; Roche) and phosphatase inhibitor cocktail 1 (Sigma-Aldrich) using mortar and pestle. Twenty micrograms of protein homogenate was separated by SDS-PAGE and transferred to nitrocellulose membranes. Primary antibodies were diluted in blocking solution as follows: rabbit Shv, 1:500; anti-tubulin 1:500 (7E10, DSHB); anti- $\beta$ actin, 1:2500 (AC15 , Abcam); anti- $\beta$ PS integrin, 1:1500 (from R. Hynes, Massachusetts Institute of Technology, Cambridge, MA); and anti-HA, 1:200 (Santa Cruz Biotechnology).

Immunoprecipitation. One hundred fly heads were collected on dry ice and homogenized in lysis buffer (10 mM HEPES, $100 \mathrm{~mm} \mathrm{NaCl}, 10 \mathrm{~mm}$ EDTA, $1 \% \mathrm{NP}-40,1 \mathrm{~mm} \mathrm{Na}_{3} \mathrm{VO}_{4}, 50 \mathrm{~mm} \mathrm{NaF}$, and $250 \mathrm{~nm}$ cyclosporin A) supplemented with complete EDTA-free protease inhibitor cocktail tablets (Roche). Anti-HA-agarose beads ( $20 \mu$ l; Sigma-Aldrich) were added to the extracts and rotated at $4^{\circ} \mathrm{C}$ overnight for immunoprecipitation. After washing three times with lysis buffer, the immunocomplexes were eluted with SDS sample buffer, and all of the eluates were used for Western blotting.

Protein purification and pull-down experiments. Protein purification was conducted as previously described (Chen et al., 2014). Briefly, His and HA doubly tagged Shv or Shv mutant (His-Shv-HA or His-Shv ${ }^{\text {LNV }}$ HA) were obtained by cloning the sequences of Shv or Shv ${ }^{\text {LNV }}$ into pET15b vector (Novagen). The BL21 strain containing the expression plasmids was grown at $37^{\circ} \mathrm{C}$ until the $\mathrm{A} 600$ of the culture reached $0.6-$ 0.8 . The expression of the proteins was induced by $1.0 \mathrm{~mm}$ isopropyl $\beta$-D-1-thiogalactopyranoside for $4 \mathrm{~h}$ at $30^{\circ} \mathrm{C}$, and bacteria were harvested and stored at $-80^{\circ} \mathrm{C}$. The Ni-NTA Purification System (Invitrogen) was used to purify His-tagged proteins. Eluted His-Shv-HA and His$\mathrm{Shv}^{\mathrm{LNV}}$-HA were dialyzed into PBS using Slide-A-Lyzer Dialysis (ThermoScientific) and concentrated using a Vivaspin Centrifugal Concentrator (Vivaproducts). For the pull-down experiments, His-Shv-HA or HisShv ${ }^{\mathrm{LNV}}$-HA containing bacterial cell lysates was incubated with antiHA-agarose beads (Sigma-Aldrich) at $4^{\circ} \mathrm{C}$ overnight and washed with lysis buffer four times. Fly protein ( $2.5 \mathrm{mg}$ from wild-type flies) were precleared with anti-HA-agarose beads for $1 \mathrm{~h}$, then incubated with antiHA-agarose beads coupled with Shv or Shv ${ }^{\mathrm{LNV}}$ at $4^{\circ} \mathrm{C}$ for $3 \mathrm{~h}$, washed with lysis buffer for 4 times, and eluted with SDS-PAGE sample buffer. All of the eluates were used for Western blotting.

Statistics. All data are presented as the mean \pm SEM. Sample numbers are shown in the graphs or figure legends and represent biological replicates. The number of samples used is consistent with established standards in the literature. Samples were randomized during dissection, image collection, and data analyses to minimize bias. For paired samples, Student's $t$ test was used. For multiple samples, one-way ANOVA followed by post hoc analysis with Bonferroni's multiple-comparison test was used to determine statistical significance.

\section{Results}

\section{Shriveled is a presynaptically secreted protein that modulates synaptic growth}

We previously characterized a sterile fly mutant, which we named shriveled (shv) due to the appearance of shriveled testes (Lee et al., 2016). Our results revealed that Shv is a secreted protein that activates $\beta$ PS integrin in the fly testes to maintain niche architecture (Lee et al., 2016). We also found that Shv protein is expressed in the Drosophila nervous system and present in the larval motor neurons but is absent in $s h v^{1}$, a P-element insertion mutant with only $\sim 3 \%$ of the $s h v$ transcript as the wild type (Lee et al., 2016; Fig. $1 A, B)$. Immunostaining of the larval brain revealed that the majority of Shv-positive cells colocalized with Elav-positive neurons in the ventral ganglion, and Shv is present only at low levels in a minor subset of Repo-positive glial cells (Fig. $1 B$, arrowhead). We therefore investigated whether Shv is present locally at the NMJ. Immunostaining using nonpermeabilizing conditions confirmed that low levels of Shv can be detected at the NMJ of wildtype larvae but not in $s h v^{1}$ mutant (Fig. 1C). The Shv protein appeared punctate and could only be observed in the NMJ using detergent-free conditions, suggesting that Shv is present extracellularly as a secreted protein. We also generated transgenic flies with the full-length Shv protein tagged with GFP (Shv-GFP) as well as Shv with truncated signal peptide tagged with GFP (NoSPShv-GFP). Figure $1 D$ shows that neuronal expression of Shv-GFP leads to positive GFP signals outside of synaptic boutons but not if the signal peptide is deleted. To further differentiate the intracellular Shv-GFP signal from the extracellular Shv-GFP signal, we performed immunostaining with an anti-GFP antibody under detergent-free conditions. We found that neuronal expression of full-length Shv-GFP, but not NoSp-Shv-GFP, led to colocalization between Shv-GFP (green) and anti-GFP (red) signals (Fig. $1 D$, arrowhead), confirming secretion and the extracellular presence of Shv at the NMJ. Interestingly, when we expressed Shv-GFP or NoSp-Shv-GFP in the postsynaptic muscles using 24B-GAL4 driver (Fig. 1D), the anti-GFP antibody did not detect the extracellular Shv-GFP signal (red) in either the full-length or truncated form (despite the presence of green Shv-GFP signal in muscles), suggesting that muscles do not secrete Shv.

Having demonstrated that Shv protein is present at the NMJ, we investigated the role of Shv in regulating synaptic morphology. Immunostaining of the NMJ with HRP revealed that $s h v^{l}$ mutant showed a synapse overgrowth phenotype with an increase in the number of synaptic boutons (Fig. $2 A-C$ ). $s h v^{I}$ 
A

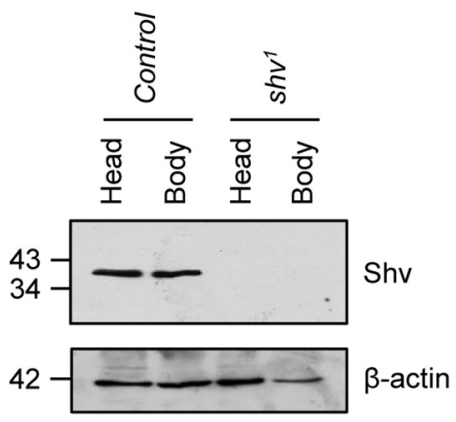

C

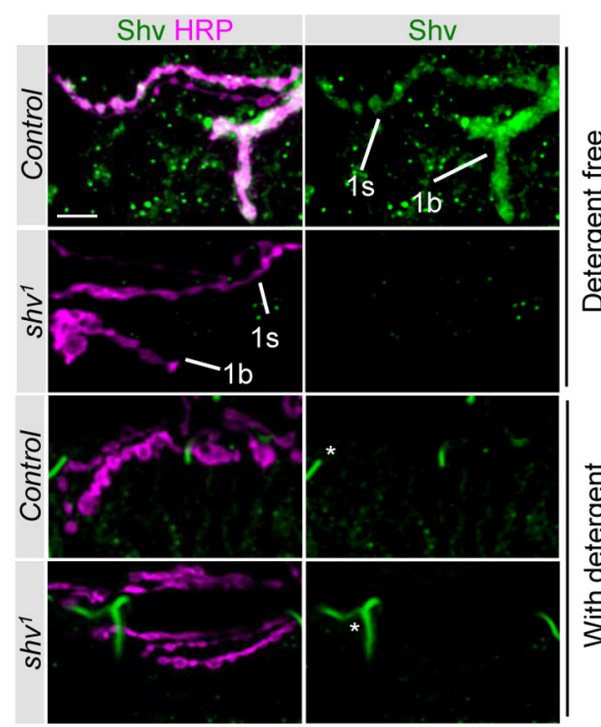

B
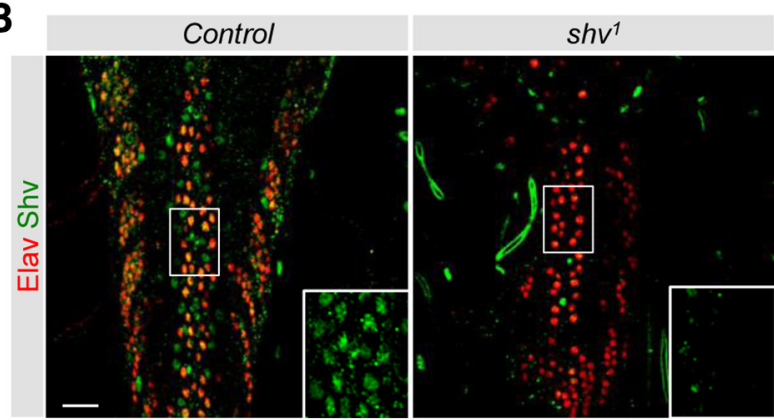

Elav Shv Repo

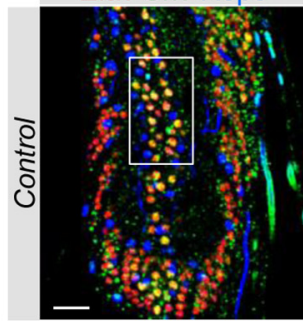

Shv

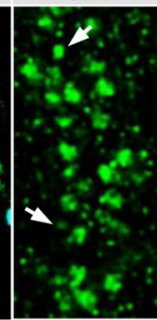

Elav Shv Shv Repo

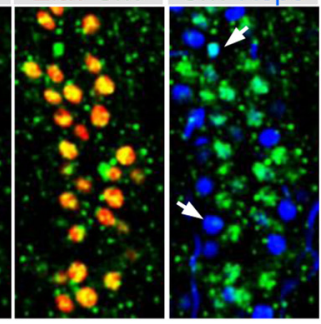

D

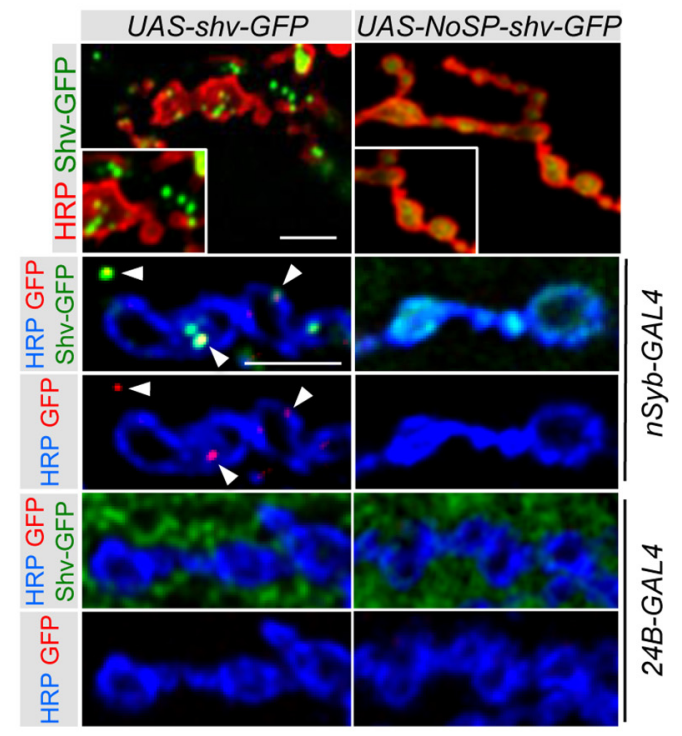

Figure 1. Shriveled is a presynaptically secreted protein. $A$, Representative Western blot showing that Shv protein is expressed in the Drosophila nervous system and shv' is virtually a null allele. $B$, Immunostaining of Drosophila larval brain of control and shv ${ }^{7}$ with indicated antibodies. Elav is a marker for neurons, and Repo is a marker for glial cells. Bottom panels show that a majority of Shv-positive cells are also Elav positive, and a minor population of Repo-positive cells is positive for Shv staining (arrow). Boxed region is enlarged, representing Shv expression in the ventral ganglion and motor neurons. Scale bar, $20 \mu \mathrm{m}$. C, Representative images of the NMJs stained with Shv antibody with or without detergent. *Background due to autofluorescence coming from tracheal branch. Scale bar, $5 \mu \mathrm{m}$. D, Images of synaptic terminal highlighting the presence of Shv-GFP outside of the boutons but not when the signal peptide has been deleted. Anti-GFP antibody (red) stained using detergent-free condition colocalizes with extracellular Shv-GFP (green) driven by pan-neuronal driver (arrowhead highlights colocalization), but not when the signal peptide was truncated. Despite the presence of Shv-GFP in muscles when Shv-GFP was expressed using 24B-GAL4 (green signal), anti-GFP antibody (red) did not detect extracellular Shv-GFP signal in full-length or truncated form. Scale bar, $5 \mu \mathrm{m}$.

mutant synapses also displayed an elevated number of "ghost boutons," which are undifferentiated boutons that are immature and have not yet been stabilized (Fig. $2 D, E$ ). These ghost boutons contain synaptic vesicles and express the neuronal membrane marker recognized by anti-HRP antibody but lack active zones or postsynaptic Dlg (Ataman et al., 2008; Vasin et al., 2014). The observation that there are more ghost boutons as well as an overall increase in synaptic complexity further suggests that the presence of Shv is required to restrict synaptic growth.

We next tested whether the presynaptic and/or the postsynaptic source of Shv is important for modulating synaptic growth. To this end, we knocked down Shv using shv-RNAi, which re- duced Shv protein level to $0.14 \pm 0.05$-fold of control $(n=4$; Fig. $2 C)$. Driving $s h v$-RNAi using a pan-neuronal driver, $n S y b-G A L 4$, caused a synaptic overgrowth phenotype and an accumulation of immature ghost boutons, but not when $s h v-R N A i$ was expressed in muscles using the 24B-GAL4 driver (Fig. 2). In addition, neuronal expression of full-length Shv sufficiently rescued the synaptic phenotypes of $\operatorname{sh} v^{1}$ but not when Shv was expressed in muscle. Deleting the signal peptide of Shv abolished the ability of neuronal Shv to rescue $s h v^{1}$ phenotype. Together, these results indicate that the release of Shv from neurons is necessary for normal synaptic growth, likely because neurons have the proper machinery to process Shv and control its release, which is consis- 
A

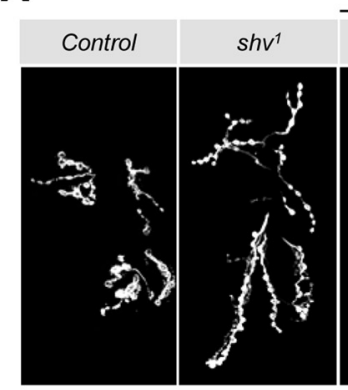

B

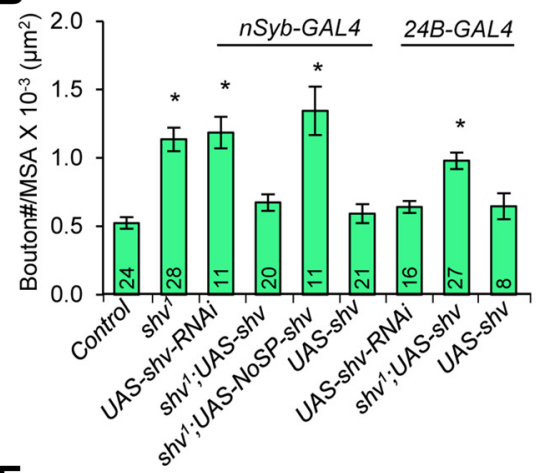

$n S y b-G A L 4$

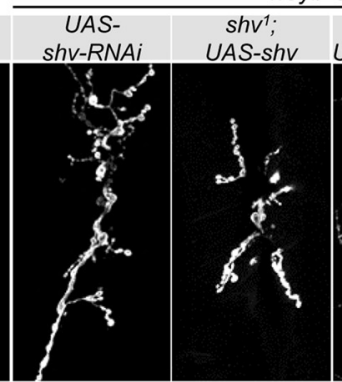

C

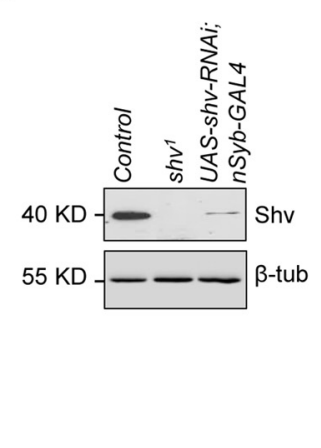

24B-GAL4

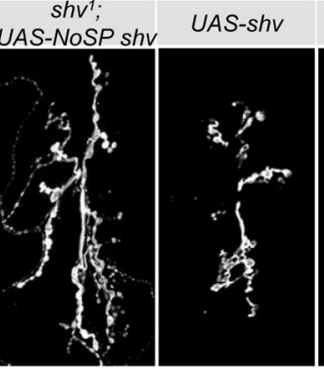

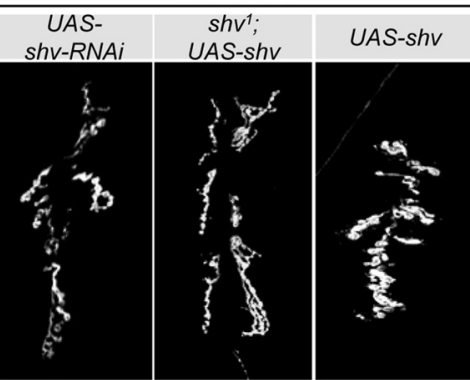

D

E

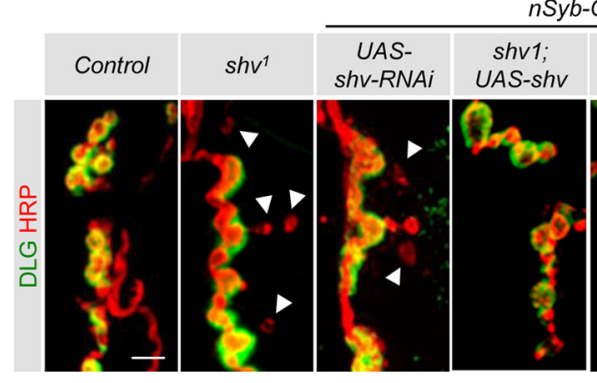

$n S y b-G A L 4$

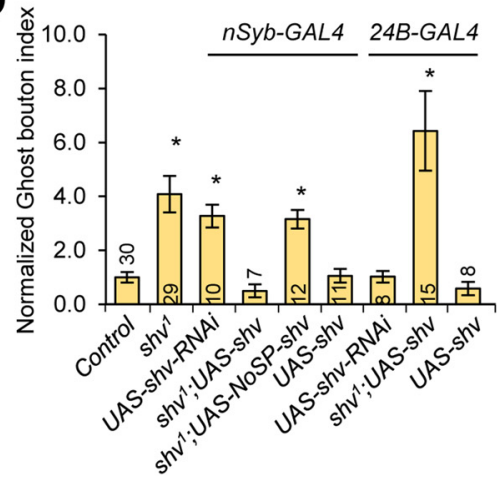

24B-GAL4
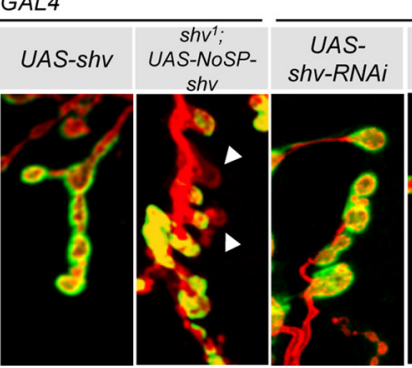

shv1;
UAS-sh

UAS-shv
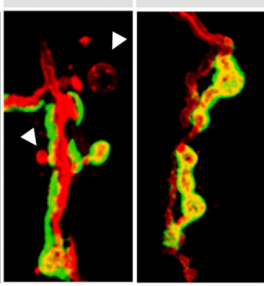

Figure 2. Shriveled modulates synaptic growth. $\boldsymbol{A}$, Muscle area 6/7 NMJ at A2 stained by HRP for the indicated genotypes. Scale bar, $10 \mu \mathrm{m}$. $\boldsymbol{B}$, Number of boutons normalized to muscle surface area (MSA). C, Representative Western blot showing relative levels of Shv protein in protein extracts of dissected larval brains. $\boldsymbol{D}$, Relative ghost bouton index across genotypes. $\boldsymbol{E}$, Images of NMJs labeled with the indicated antibodies. Arrowheads point to ghost boutons that are recognized by HRP labeling while lacking Dlg immunostaining. Scale bar, $5 \mu \mathrm{m}$. ${ }^{*} p<0.05$ compared with control. All values represent the mean \pm SEM.

tent with our findings in Figure $1 D$. We also found that the overexpression of $s h v$ alone did not affect synaptic growth and complexity (Fig. 2), suggesting that even though the presence of $\mathrm{Shv}$ is required to restrict synaptic growth, it does not inhibit synaptic growth.

\section{Shv modulates integrin receptor activation at the NMJ}

Since our previous study in fly testes and S2 cells demonstrated that Shv can activate $\beta$ PS integrin signaling (Lee et al., 2016), we therefore tested whether Shv acts as a novel regulator of integrin signaling at the NMJ. First, we assayed whether Shv can physically interact with $\beta$ PS integrin receptor (Fig. $3 A$ ). To this end, we immunoprecipitated Shv from the protein extract of UAS-shv transgenic flies driven by the $n S y b$-GAL4 driver, which expresses Shv tagged with HA (Shv-HA), and interaction between Shv and $\beta$ PS integrin was confirmed by immunoblotting. Transgenic flies without the driver were used as a negative control since it does not express Shv-HA protein. Indeed, we confirmed that Shv interacts with $\beta$ PS integrin (Fig. 3A). Next, we asked whether (1) Shv indeed modulates integrin signaling at the NMJ, and (2) whether Shv genetically interacts with $\beta$ PS integrin receptor to regulate synaptic growth. As a measure of integrin activation, we determined the levels of phosphorylated focal adhesion kinase (pFAK), since its level strongly correlates with integrin activation (Mitra et al., 2005; Tsai et al., 2008; Harburger and Calderwood, 2009; Campbell and Humphries, 2011). As $\beta$ PS integrin receptors are present presynaptically and postsynaptically (Beumer et al., 1999), we monitored the levels of pFAK in the presynaptic terminal (within the boundary of neuronal membrane marker HRP) and the total levels at the synapse (within the boundary of postsynaptic marker Dlg; Fig. $3 B$ ). We noticed that pFAK staining appeared punctate both within and outside of areas demarcated by HRP and, thus, further examined the relative localization of pFAK signals. To this end, we performed coimmunostaining with NC82, an active zone marker (Wagh et al., 2006), as well as used phalloidin to outline the postsynaptic muscles (Hunt and Demontis, 2013). As shown in Figure 3C, punctate presynaptic pFAK spots (area demarcated by HRP) are not concentrated at the active zone, as there is only minimal overlap between pFAK and NC82 signals. Furthermore, although we detected pFAK staining throughout the muscle, the majority of the pFAK signal is enriched at the synapse (Fig. 3D), which is consistent with a previous report (Tsai et al., 2008). We found that, consistent with Shv being an activator of integrin signaling, depleting Shv in $s h v^{I}$ 
A

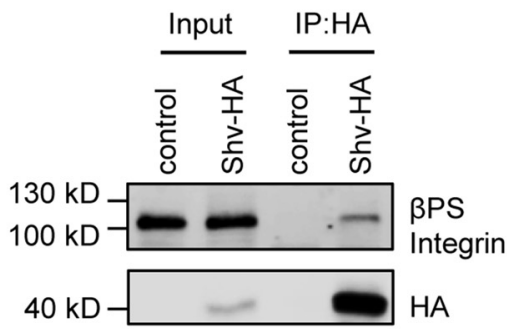

C

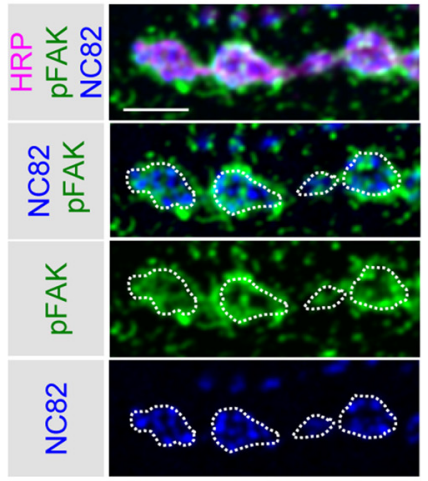

B

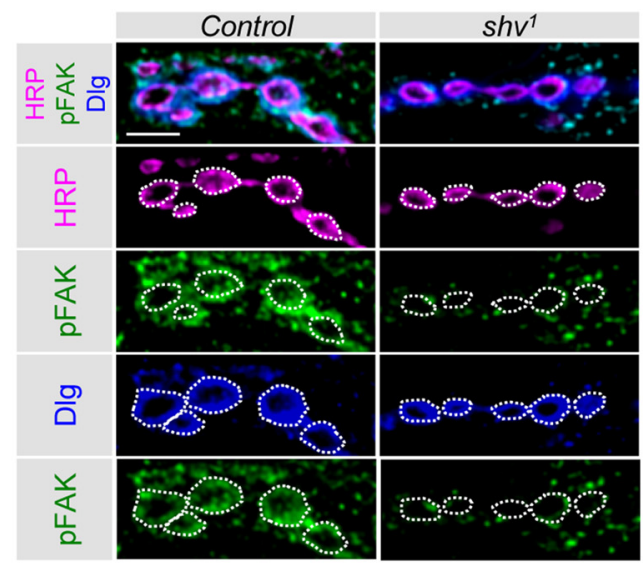

D

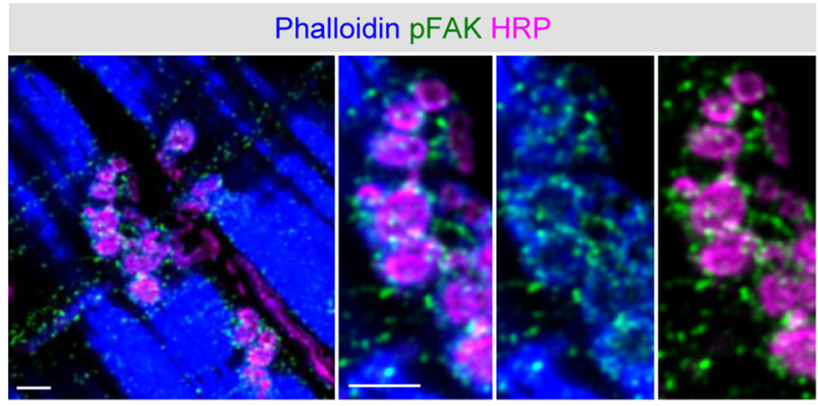

$\mathbf{E}$

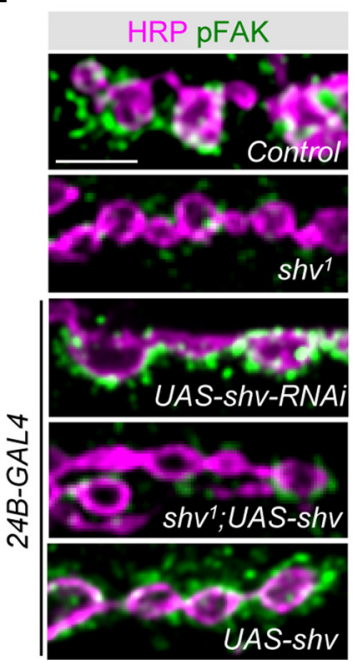

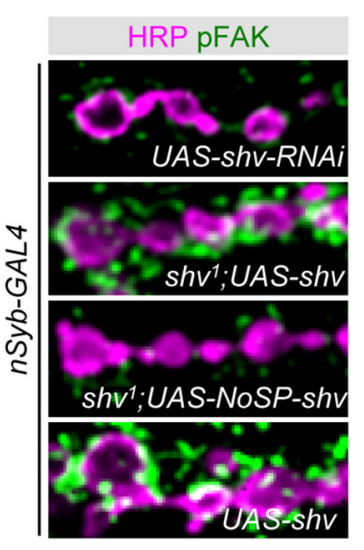

$\mathbf{F}$

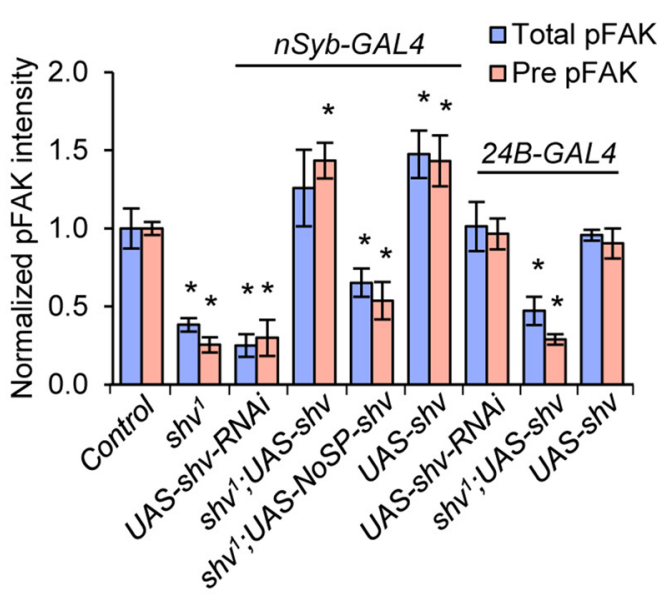

Figure 3. Shv activates integrin receptor at NMJ. A, Immunoprecipitation (IP) assay followed by Western blots with the indicated antibodies demonstrate interaction between Shv and $\beta$ PS integrin. The control used is transgenic flies without the nSyb-GAL4 driver; thus, it does not express Shv-HA. B, The presynaptic pFAK signal was calculated by outlining the presynaptic bouton using the HRP signal, whereas total pFAK signal was determined by outlining the postsynaptic area using Dlg staining. C, Wild-type NMJs stained with the indicated antibodies. Presynaptic membrane is demarcated by HRP staining (white outline). The pFAK signal is not enriched at the active zone as detected by NC82. D, Phalloidin staining highlights the postsynaptic muscle of a wild-type NMJ. pFAK signal can be seen throughout the muscle but is enriched at the synapse and is present both presynaptically and postsynaptically. HRP highlights the presynaptic bouton, and some phalloidin staining, which detects F-actin, is also enriched at the postsynaptic bouton. $E$, Representative images of NMJs stained with HRP and pFAK across genotypes. $F$, Quantification of presynaptic pFAK and total pFAK levels at synaptic terminal by outlining HRP or Dlg, respectively. Scale bars, $5 \mu \mathrm{m} .{ }^{*} p<0.05$ compared with control. All values represent the mean \pm SEM.

or neuronal knockdown of Shv using RNAi both led to decreased pFAK levels presynaptically, as well as to decreased total levels of pFAK at the NMJ (Fig. 3E, F; Table 1). Neuronal overexpression of shv also elevated both presynaptic and total pFAK levels at the NMJ compared with control, but not when shv is overexpressed in muscles using the $24 B-G A L 4$ driver. These results indicate that the release of Shv by neurons activates integrin signaling presynaptically and postsynaptically in an autocrine and paracrine fashion. In support of this, neuronal expression of the full-length Shv restored pFAK level of $s h v^{I}$ both presynaptically and postsynap- 
Table 1. Sample number used in Figures $3 E$ and $4 E$

\begin{tabular}{|c|c|c|}
\hline Genotype & TotalpFAK & Pre-pFAK \\
\hline Control & 77 & 61 \\
\hline$s h v^{1}$ & 23 & 9 \\
\hline UAS-shv-RNAi/nSyb-GAL4 & 7 & 6 \\
\hline shv ${ }^{1} ;$ UAS-shv/nSyb-GAL4 & 10 & 9 \\
\hline shv ${ }^{1} ;$ UAS-NoSP-shv/nSyb-GAL4 & 11 & 12 \\
\hline UAS-shv/nSyb-GAL4 & 8 & 7 \\
\hline UAS-shv-RNAi/24B-GAL4 & 8 & 8 \\
\hline $\operatorname{shv}^{1} ;$ UAS-shv/24B-GAL4 & 11 & 12 \\
\hline UAS-shv/24B-GAL4 & 8 & 8 \\
\hline$m_{y s} s^{t s 1}$ & 7 & 6 \\
\hline mys $^{\text {ts }} ;$ shv $^{1}$ & 7 & 7 \\
\hline mys ${ }^{t s 1} ;$ UAS-shv/nSyb-GAL4 & 14 & 13 \\
\hline shv ${ }^{7} ;$ UAS-mys/nSyb-GAL4 & 10 & 10 \\
\hline UAS-mys/nSyb-GAL4 & 6 & 6 \\
\hline shv ${ }^{1} ;$ UAS-mys/24B-GAL4 & 17 & 13 \\
\hline UAS-mys/24B-GAL4 & 10 & 10 \\
\hline mys $^{\text {ts }} ;$;UAS-shv/24B-GAL4 & 12 & 12 \\
\hline
\end{tabular}

tically, whereas the NoSP-Shv did not (Fig. 3E,F). This suggests that $S h v$ activates integrin signaling via an outside-in mechanism, consistent with previous experiments performed using S2 cultures (Lee et al., 2016).

Next, we investigated whether Shv interacts with $\beta$ PS integrin to modulate integrin signaling and synaptic growth. We found that $\beta$ PS hypomorphic mutant, $m y s^{t s l}$, displayed an increase in bouton number and ghost bouton index similar to that of $s h v^{1}$ (Fig. $4 A-D$ ). Like $s h v^{1}$, we also saw a corresponding decrease in the levels of presynaptic and total pFAK in the $m y s^{t s l}$ mutant (Fig. $4 E, F)$. This result confirms reduced integrin activation both presynaptically and postsynaptically in the $m y s^{t s l}$ mutant, consistent with the known distribution of $\beta$ PS integrin at the NMJ (Beumer et al., 1999). Note that because the mys-null allele is embryonic lethal, we used $m y s^{t s 1}$, a strong hypomorphic allele of $m y s$ that shows substantially reduced Mys protein level at $25^{\circ} \mathrm{C}$ (Beumer et al., 1999). To further confirm genetic interaction between Shv and $\beta$ PS integrin, we examined double mutants of $m y s^{t s 1}$ and $s h v^{l}$. We found that $m y s^{t s l} ; s h v^{1}$ double mutants displayed the same phenotypes as either mutant alone in synaptic morphology and pFAK levels (Fig. 4A-F; Table 1), confirming that they act in the same genetic pathway. In addition, $m y s^{t s 1}$ hypomorphic allele dampened the effect of $s h v$ overexpression on pFAK, suggesting that integrin acts downstream of Shv. Upregulating Shv in neurons of $m y s^{t s l}$ hypomorphic mutant also partially alleviated the synaptic overgrowth phenotype and corrected pFAK levels of $m y s^{t s 1}$ (Fig. 4), likely because the extra amount of Shv present at the synapse enhances signaling through the remaining $\beta$ PS integrin receptors in $m y s^{t s 1}$. On the other hand, upregulating Shv in muscles of $m y s^{t s 1}$ mutant did not restore the synaptic overgrowth phenotype or the pFAK levels (Fig. 4), further supporting the finding that postsynaptic muscles do not release Shv extracellularly (Fig. 1D). We also attempted to restore integrin signaling by overexpressing mys (UAS-mys) in the neurons or muscles of $s h v^{I}$. However, both failed to restore the synaptic overgrowth phenotype of $s h v^{1}$ (Fig. 4A,B), raising the possibility that the presence of $\mathrm{Shv}$ is required for integrin activation. Indeed, pFAK staining revealed that upregulation of the integrin receptors alone failed to restore integrin signaling in the absence of an activator, Shv (Fig. $4 E, F)$. However, in the presence of Shv (wild-type background), overexpression of mys in neurons and muscles increased the corresponding level of presynaptic pFAK and total pFAK, respectively, confirming that the presence of Shv is necessary for efficient integrin signaling. Together, these results indicate that activation of integrin receptors by extracellular Shv restricts synaptic growth.

\section{Shv is released during intense activity to acutely activate integrin signaling}

In addition to modulating synaptic growth during development, integrin receptors also contribute to activity-dependent synaptic plasticity (Rohrbough et al., 2000; Tsai et al., 2012a; Dani et al., 2014). Having demonstrated that Shv is a secreted protein that activates integrin signaling, we next asked whether Shv release is regulated by activity. First, we used an established spaced depolarization protocol with three pulses of high $\mathrm{KCl}$ that has been shown to induce new bouton formation (Vasin et al., 2014). Consistent with the study by Vasin et al. (2014), three pulses of high $\mathrm{KCl}$ increased the number of ghost boutons (Fig. 5A); however, there was no detectable change in Shv level at the NMJ, and pFAK level was significantly reduced (Fig. $5 B-E$ ). We also tested another spaced depolarization protocol that can more robustly induce new bouton formation ( $5 \times$ high KCl; Ataman et al., 2008). Despite a significant increase in ghost bouton numbers following $5 \times$ pulse stimulation (Fig. $5 F$ ), this spaced stimulation protocol again failed to elevate the Shv level and resulted in reduced pFAK at the synapse (Fig. 5G-J). These results are consistent with previous findings that activity-induced synaptic growth and elongation downregulates integrin signaling, presumably to minimize the opposing adhesive forces generated by integrin activation at the presynaptic and postsynaptic terminals (Tsai et al., 2012a; Dani et al., 2014).

Next, we tested whether Shv is instead released in response to persistent neuronal activity by stimulating wild-type larval prep with high $\mathrm{KCl}$ for $10 \mathrm{~min}$. Figure $6 \mathrm{~A}$ demonstrates that this persistent stimulation protocol led to extensive upregulation of the Shv signal at the NMJ detected using nonpermeabilizing conditions that were not observed in $\operatorname{sh} v^{1}$ mutant, suggesting that Shv is released extracellularly at the synapse. To further examine whether Shv is specifically released in response to high synaptic demand, we monitored extracellular Shv signal in response to nerve stimulation at high or low frequencies. Figure $6 \mathrm{~B}$ demonstrates that Shv is detected at the NMJ following $10 \mathrm{~Hz}$ stimulation, but not $1 \mathrm{~Hz}$, revealing that Shv is selectively released by intense synaptic activity. Enhanced Shv release by prolonged high $\mathrm{K}^{+}$is accompanied by greater integrin activation as measured by the increase in either presynaptic or total pFAK levels, but not in $s h v^{l}$ mutant or $m y s^{t s 1}$ mutants (Fig. 6C,D). High-frequency nerve stimulation, but not low-frequency nerve stimulation, also elevated pFAK levels in control larvae. Again, such a change in pFAK was not detected in $s h v^{1}$ or $m y s^{t s 1}$ following high-frequency nerve stimulation (Fig. $6 E, F$ ), implying that only intense neuronal activity triggers $S h v$ release and the activation of integrin signaling.

We also tested the involvement of another integrin subunit, $\beta_{\nu}$ integrin, which is present presynaptically at the Drosophila NMJ and has been shown to affect pFAK levels and synaptic growth via retrograde laminin signaling (Tsai et al., 2008, 2012a). We found that, consistent with a previous report, the knockdown of $\beta_{\nu}$ integrin in neurons generated synaptic overgrowth (Fig. 6G) and decreased presynaptic pFAK levels (Fig. $6 H, I)$, producing phenotypes similar to $s h v^{1}$ and $m y s^{t s 1}$. However, unlike $s h v^{1}$ or $m y s^{t s I}$ mutants, intense activity still triggered a presynaptic increase in pFAK levels in NMJ with neuronal $\beta_{\nu}$ integrin knockdown despite a lower initial pFAK 
A

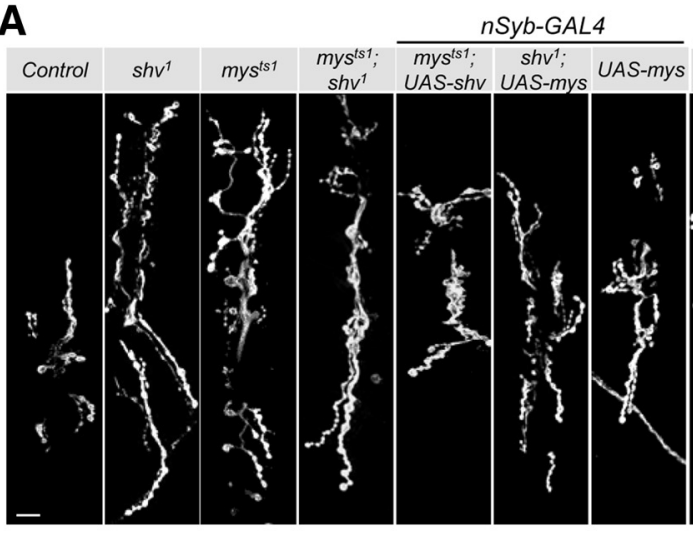

24B-GAL4

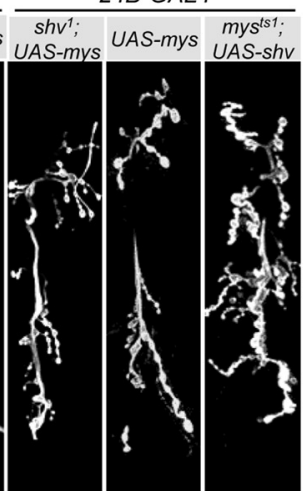

B

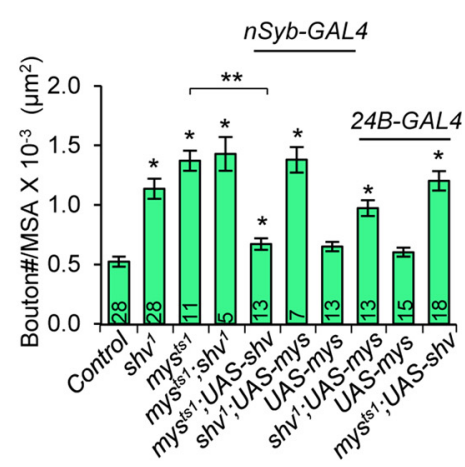

C

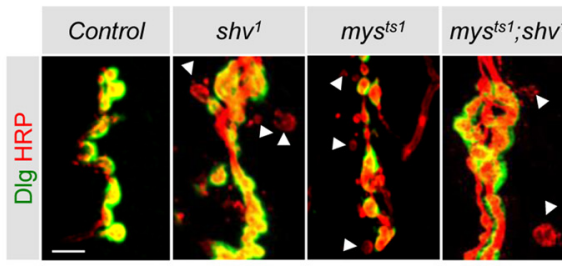

D
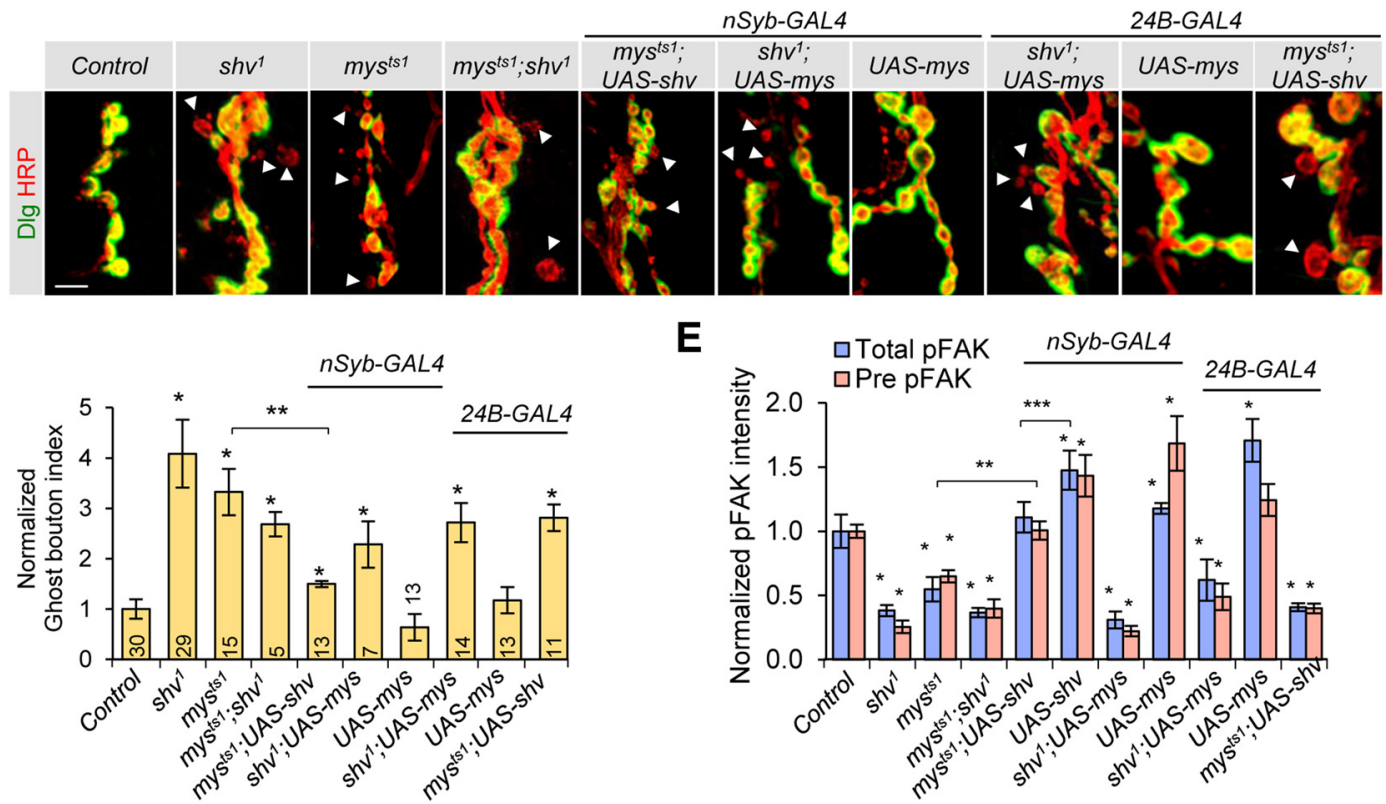

E

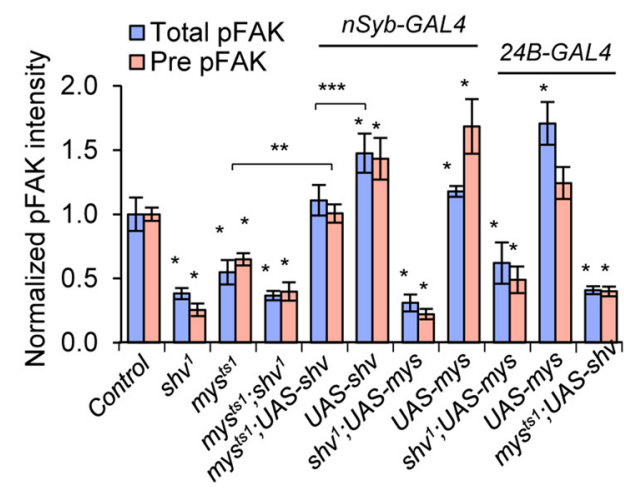

$\mathbf{F}$

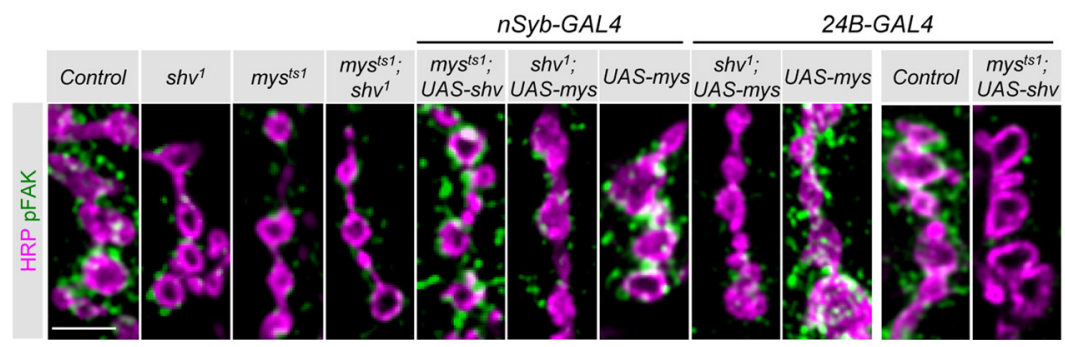

Figure 4. Shv genetically interacts with integrin. $A$, Representative images of NMJs stained with HRP for the indicated genotypes. Scale bar, $10 \mu \mathrm{m} . \boldsymbol{B}$, Quantification of bouton numbers normalized to muscle surface area (MSA). C, Images of NMJs labeled with HRP and Dlg. Arrowheads point to ghost boutons that are HRP positive but Dlg negative. Scale bar, $5 \mu \mathrm{m}$. D, Quantification of relative ghost bouton index. E, Quantification of presynaptic pFAK and total pFAK levels at synaptic terminal by outlining HRP or Dlg, respectively. $F$, Representative images of NMJs stained with HRP and pFAK across genotypes. Scale bar, $5 \mu \mathrm{m} .{ }^{*} p<0.05$ compared with control; ${ }^{* *} p<0.05$ compared with indicated genotypes; ${ }^{* * *} p<0.05$ when comparing the value of total $p F A K$ between the indicated genotypes. All values represent the mean \pm SEM.

level, further suggesting that strong activity mainly triggers $\beta P S$ integrin activation independent of $\beta_{\nu}$ integrin receptors and retrograde laminin signaling (Fig. $6 H, I$ ). Together, these data support the claim that activity-dependent Shv secretion acts to acutely activate $\beta$ PS integrin signaling.

\section{Activation by Shv during intense activity triggers} synapse maturation

What is the physiological consequence of activity-dependent Shv secretion and the activation of integrin receptors? We hypothesized that while mild or patterned synaptic activity triggers syn- aptic growth, intense neuronal activity leads to Shv release to acutely activate integrin and promote synaptic bouton maturation. Similar to spaced $\mathrm{K}^{+}$stimulation, prolonged high $\mathrm{K}^{+}$stimulation paradigm triggered an increase in the number of ghost boutons in control larvae (Fig. $7 A, B$ ). Unstimulated $\operatorname{sh} v^{1}$ and $m y s^{t s 1}$ both started with a higher number of ghost boutons comparable to the stimulated control, and the number of ghost boutons increased slightly (albeit not significantly) upon stimulation, perhaps because bouton budding reached a threshold. Interestingly, this stimulation paradigm also acutely increased the size of synaptic boutons (Fig. $7 C, D$ ), suggesting that prolonged stimu- 
A

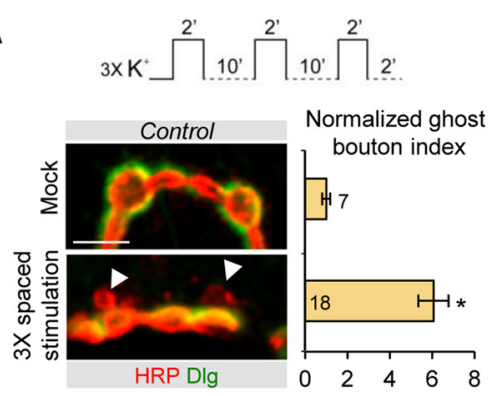

D

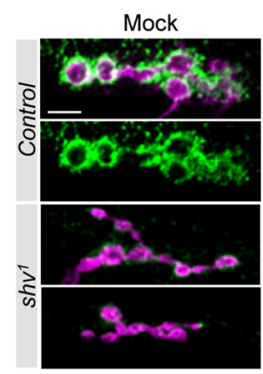

G

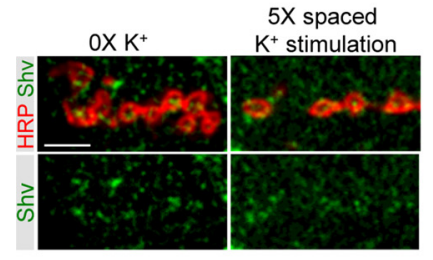

B

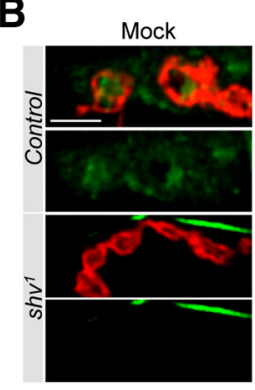

E $\mathrm{K}^{+}$Stimulation

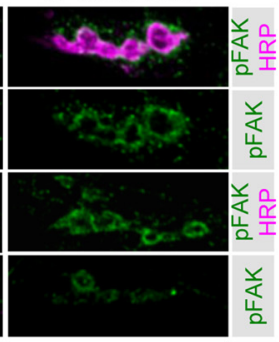

H

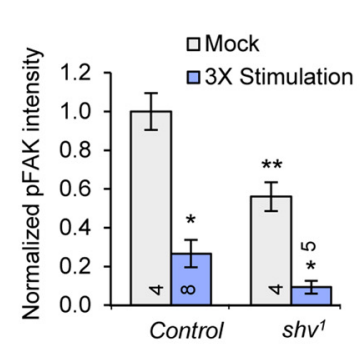

I

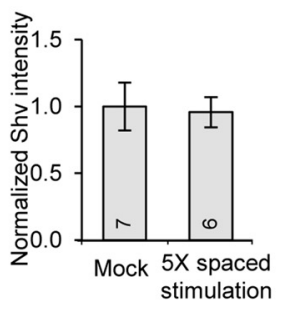

C

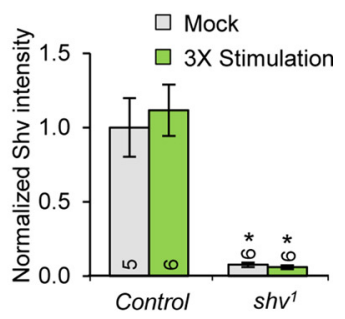

F

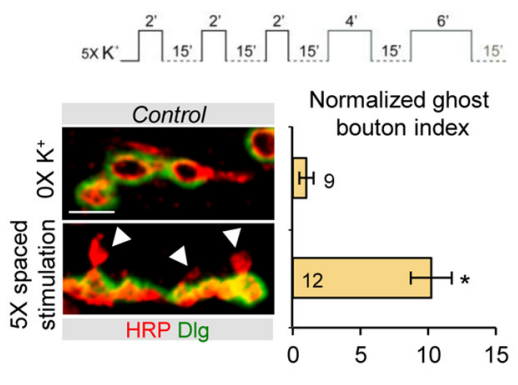

J
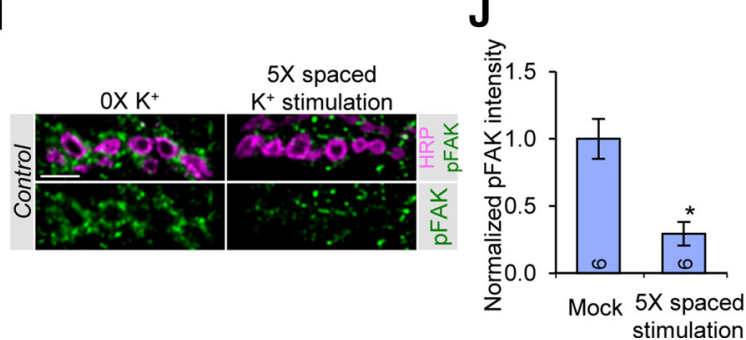

Figure 5. Pulsed high $\mathrm{K}^{+}$stimulation did not trigger Shv release or change in pFAK levels. $\boldsymbol{A}, \boldsymbol{F}$, Representative images and quantification of ghost boutons after $3 \times$ spaced high $\mathrm{K}^{+}$ stimulation $(\boldsymbol{A})$ or $5 \times$ spaced high $\mathrm{K}^{+}$stimulation $(\boldsymbol{F})$. Arrowheads highlight the ghost boutons. $\boldsymbol{B}, \mathbf{G}$, Representative images of Shv at the NMJs following $3 \times$ spaced high $\mathrm{K}^{+}$ stimulation $(\boldsymbol{B})$ or $5 \times$ spaced high $\mathrm{K}^{+}$stimulation $(\boldsymbol{G})$, which were stained using detergent-free condition. $\boldsymbol{C}, \boldsymbol{H}$, Quantification of relative Shv intensity. $\boldsymbol{D}, \boldsymbol{I}$, Images of NMJ stained with pFAK and HRP antibodies following stimulation with the indicated conditions. $\boldsymbol{E}, \boldsymbol{J}$, Quantification of pFAK levels upon $3 \times$ spaced K $^{+}$stimulation $(\boldsymbol{E})$ or $5 \times$ spaced $K^{+}$stimulation $(\boldsymbol{J})$ compared with mock treatment (without high $\mathrm{K}^{+}$). ${ }^{*} p<0.05$ when compared with mock-treated control; ${ }^{* *} p<0.05$ when comparing mock treated or $3 \times$ stimulated. Scale bars, $5 \mu \mathrm{m}$. All values represent the mean \pm SEM.

lation triggered structural modification to favor synaptic maturation. In contrast, prolonged stimulation of $s h v^{l}$ did not lead to bouton enlargement, confirming that Shv plays a crucial role in activity-dependent synaptic remodeling. Similarly, the $m y s^{t s 1}$ mutant did not expand its bouton size upon persistent stimulation (Fig. 7C,D), whereas neuronal knockdown of $\beta_{\nu}$ integrin still exhibited an increase in synaptic bouton size, which is consistent with Shv acting through a pathway independent of $\beta_{\nu}$ integrin (Fig. 7C,D). Furthermore, in accord with enhanced Shv release by high-frequency nerve stimulation, we found that intense neuronal activity selectively increased the size of synaptic boutons (Fig. $7 E, F)$. Such an increase in bouton size was not observed in control larvae under mild stimulation $(1 \mathrm{~Hz})$, or in $s h v^{1}$ and $m y s^{t s l}$ mutants under intense stimulation. Last, in agreement with hypotheses that different synaptic demand activates a distinct synaptic remodeling program and that Shv is a key player in activity-dependent synaptic remodeling, we found that the pulsed potassium stimulation paradigm, which did not trigger Shv release or elevate $\mathrm{pFAK}$, was not sufficient to increase synaptic bouton size (Fig. 7G,H).

To further confirm that changes in the size of the synaptic boutons represents activity-induced synapse maturation, we measured the levels of postsynaptic glutamate receptors. The level of GluRIII receptor, which is an essential subunit of the
Drosophila glutamate receptors at the NMJ, was monitored before and after stimulation. Following high $\mathrm{KCl}$ stimulation and high-frequency nerve stimulation, we found a significant increase in glutamate receptor abundance (GluRIII) in control NMJs (Fig. $8 A-D$ ). Interestingly, $s h v^{1}$ and $m y s^{t s 1}$ showed lower levels of glutamate receptor staining intensity before stimulation compared with control, and glutamate receptor abundance was not enhanced by neuronal activity (Fig. $8 A-D$ ). Similar results were obtained when we examined the relative abundance of GluRIIA and GluRIIB, two functionally redundant glutamate receptor subunits with distinct electrophysiological properties (Petersen et al., 1997; DiAntonio et al., 1999; Fig. 8E-G). Together, our results demonstrate that Shv and integrin activation is essential for synapse maturation during development and for activityinduced synapse remodeling and maturation.

As we have shown that Shv activates $\beta$ PS integrin receptors bidirectionally, we next investigated the requirement of presynaptic and postsynaptic $\beta$ PS integrin receptors in regulating activity-induced changes in bouton size and glutamate receptor abundance. To this end, we knocked down mys presynaptically or postsynaptically using tissue-specific drivers and a $m y s-R N A i$ line previously shown to effectively reduce $\beta$ PS integrin expression (Okumura et al., 2014). Surprisingly, even though presynaptic knockdown of mys reduced the basal levels of GluRIII, it did not 
A


E

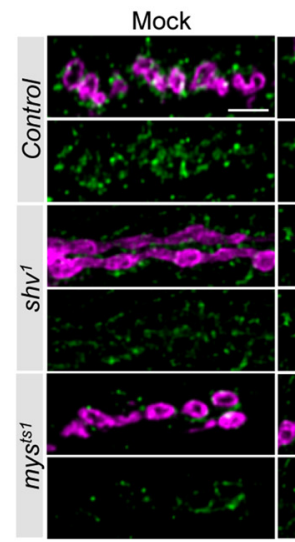

$5 \min @ 1 \mathrm{~Hz}$

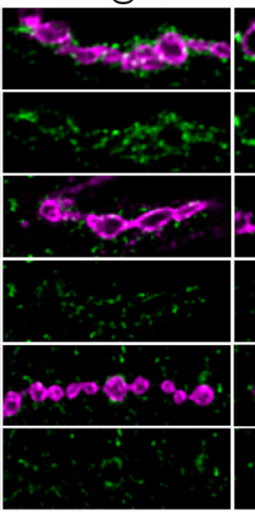

$5 \min @ 10 \mathrm{~Hz}$

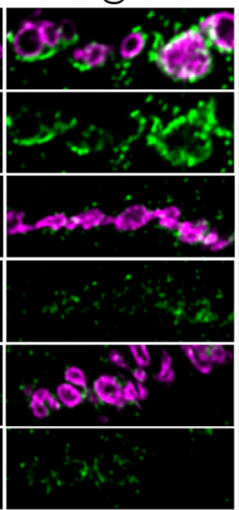

H

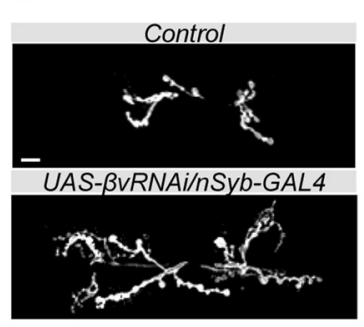

B

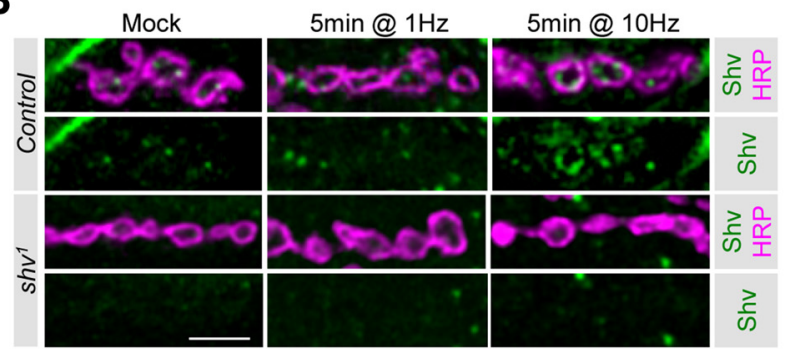

D

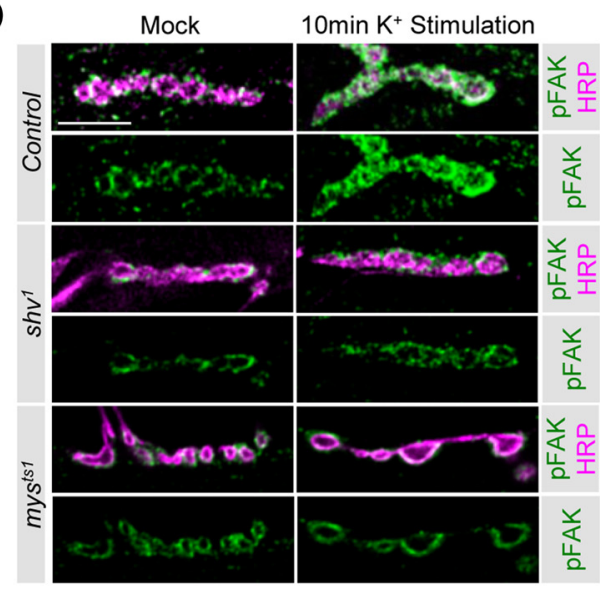

F

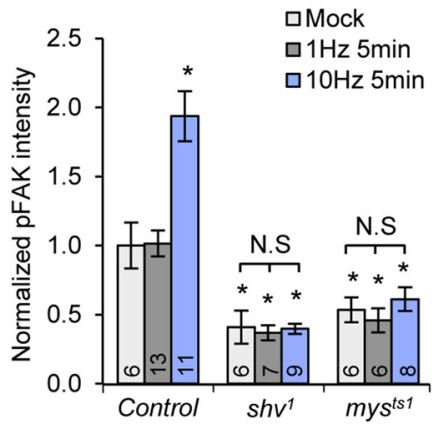

I

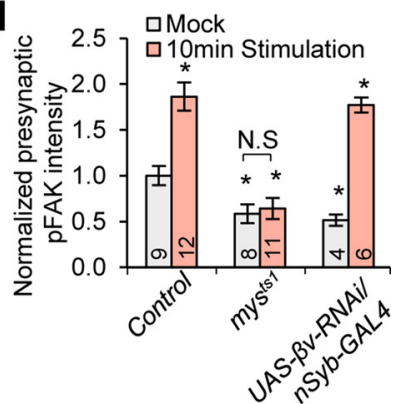

Figure 6. Shv is secreted during intense synaptic stimulation to acutely activate integrin signaling. $A$, Representative images of NMJ stimulated with high $\mathrm{K}^{+}$for 10 min followed by detergent-free Shv antibody labeling. $\boldsymbol{B}$, Images representing Shv release after electrical stimulation at $10 \mathrm{~Hz}$ for $5 \mathrm{~min}$ but not by stimulation at $1 \mathrm{~Hz}$ for 5 min. Extracellular Shv was stained using detergent-free condition. C, Quantification of total and presynaptic pFAK levels before and after 10 min high K ${ }^{+}$stimulation. D, Levels of pFAKimmunostaining within NMJs treated with a persistent pulse of high $\mathrm{K}^{+}$stimulation across genotypes. $\boldsymbol{E}$, Images representing the elevated pFAK level following high-frequency electrical stimulation but not in low-frequency or shv ${ }^{7}$ and $m y s^{\text {ts }}{ }^{7}$. $\boldsymbol{F}$, Quantification of normalized total pFAK level upon high-frequency electrical stimulation. $\mathbf{G}$, Representative images showing that presynaptic knockdown of $\beta_{\nu}$ integrin results in a synaptic overgrowth phenotype. $\boldsymbol{H}$, Representative images of UAS- $\beta_{\nu} R N A i / n S y b$-GAL4 showing elevated pFAK staining after high $\mathrm{K}^{+}$stimulation for 10 min. $I$, Quantification of presynaptic pFAK levels upon high $\mathrm{K}^{+}$stimulation. Scale bars: $\boldsymbol{A}, \boldsymbol{B}, \boldsymbol{E}, \boldsymbol{H}, 5 \mu \mathrm{m} ; \boldsymbol{D}, \mathbf{G}, 10 \mu \mathrm{m} .{ }^{*} p<0.05$ compared with control. All values represent the mean \pm SEM. 
A

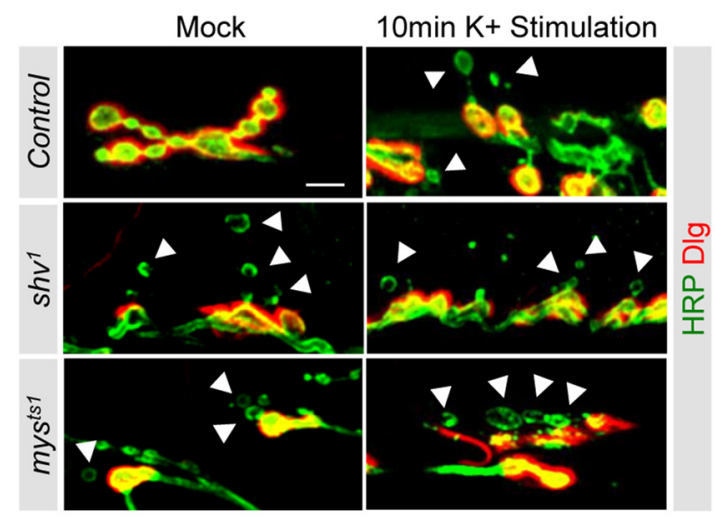

C

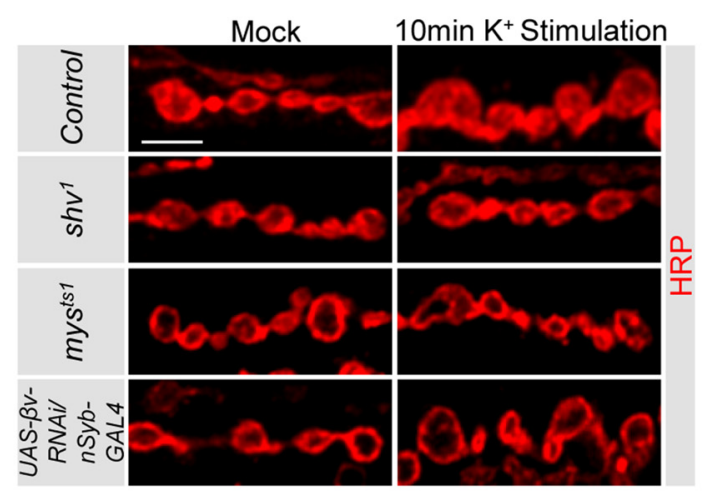

E
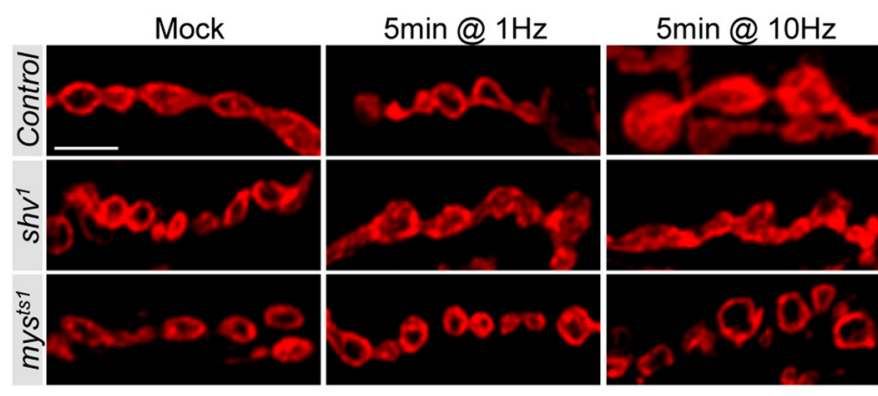

G

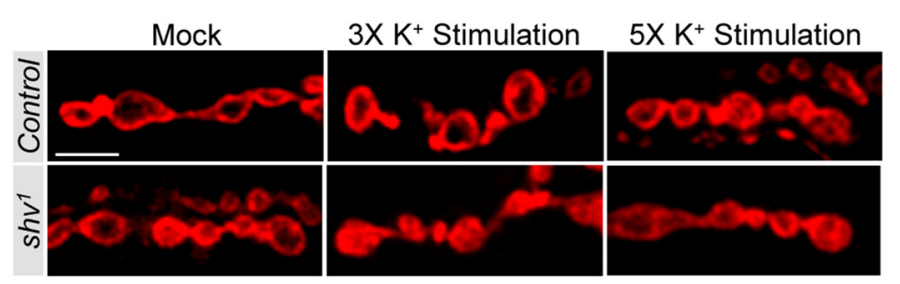

B

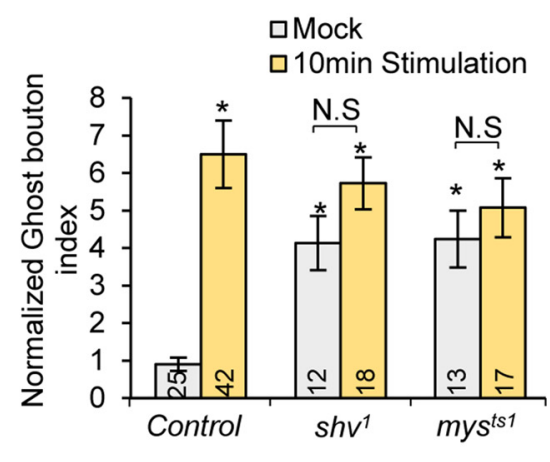

D

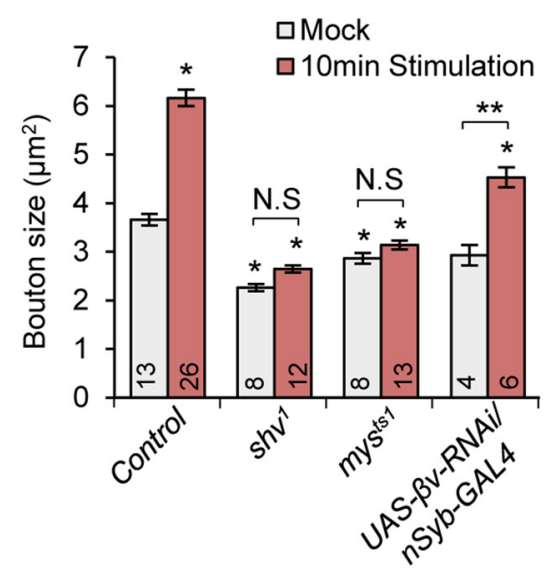

F

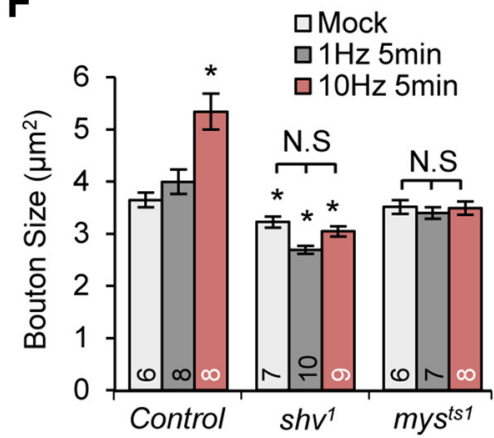

H

$\square$ Mock

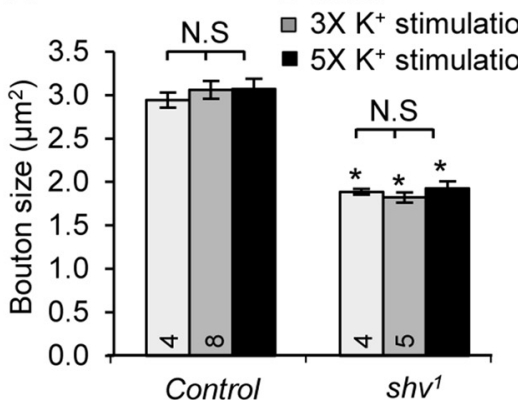

Figure 7. Shv release during intense activity induces local bouton enlargement. $A$, Representative images of ghost bouton before and after intense stimulation with high $\mathrm{K}^{+}$application (highlighted with arrowhead). $\boldsymbol{B}$, Normalized ghost bouton index present upon $10 \mathrm{~min}$ high $\mathrm{K}^{+}$depolarization. $\boldsymbol{C}$, Images representing an increase in individual synaptic bouton size after high $\mathrm{K}^{+}$ stimulation. $\boldsymbol{D}$, Quantification of the average size of type $1 \mathrm{~b}$ bouton following $10 \mathrm{~min}$ high $\mathrm{K}^{+}$application. $\boldsymbol{E}, \mathbf{G}$, Representative images of NMJ stained with HRP staining showing size of synaptic boutons following nerve-evoked stimulation $(\boldsymbol{E})$ and with and without pulses of high $\mathrm{K}^{+}$incubation $(\boldsymbol{G})$, as marked. $\boldsymbol{F}, \boldsymbol{H}$, Quantification of bouton size. Scale bars: $\boldsymbol{A}, \boldsymbol{C}, \boldsymbol{E}, \boldsymbol{G}, 5 \mu \mathrm{m}$. ${ }^{*} p<0.05$ compared with control; ${ }^{* *} p<0.05$ when comparing the indicated conditions. All values represent the mean \pm SEM. 
A

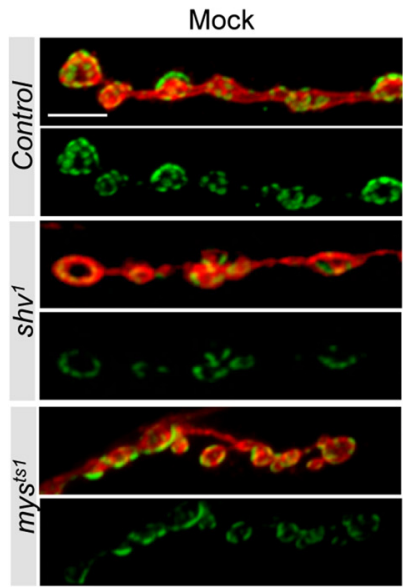

C

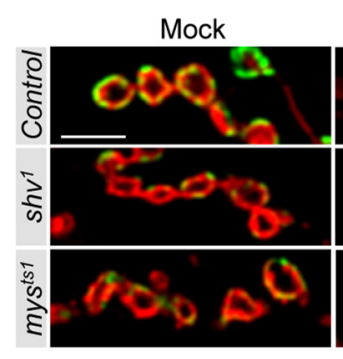

$5 \mathrm{~min} @ 1 \mathrm{~Hz}$

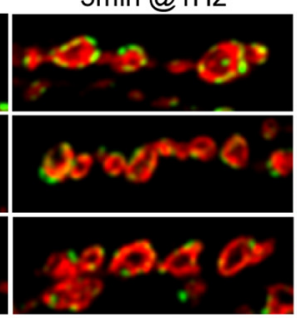

10min Stimulation



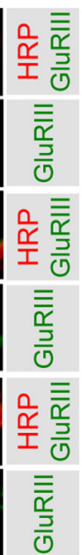

B

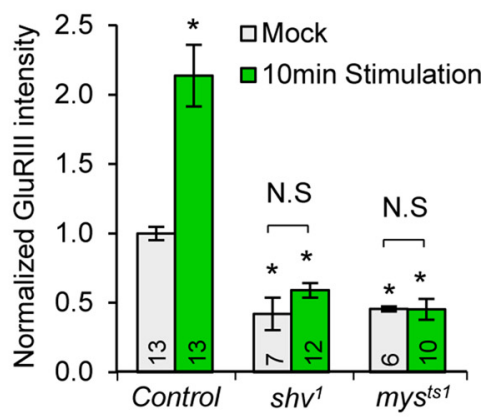

D

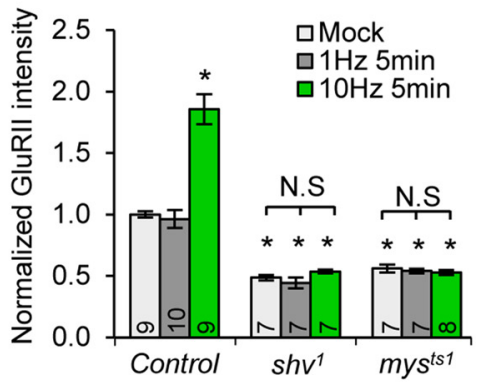

E

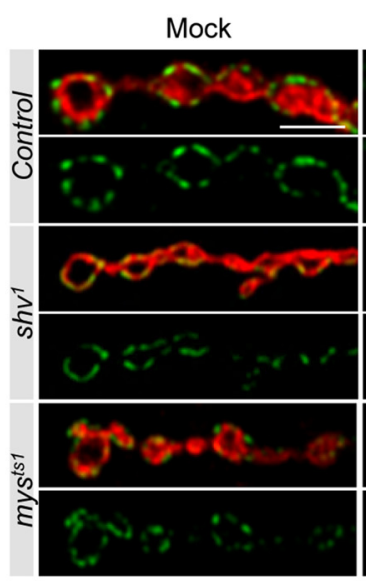

F

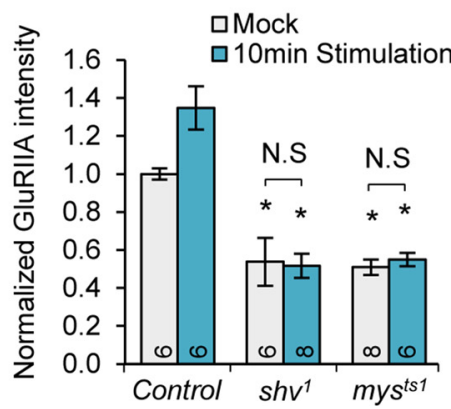

10min Stimulation

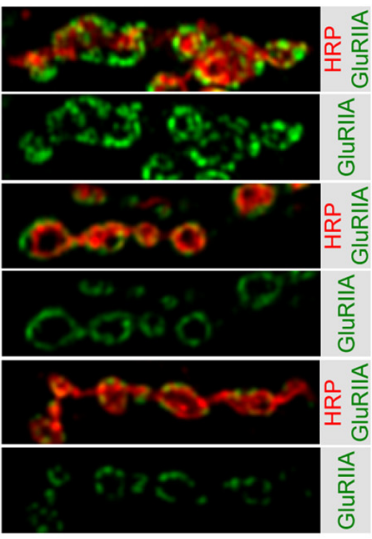

G

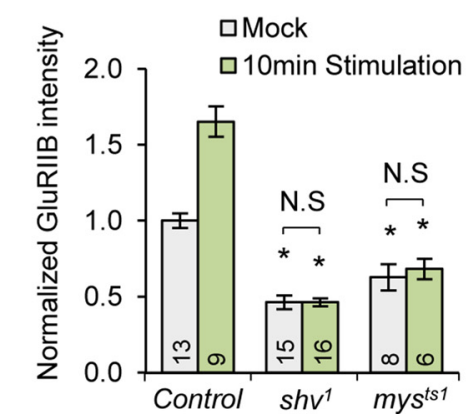

Figure 8. Release of Shv during neuronal stimulation induces synapse maturation. A, Images representing staining of GluRIII at the NMJ with or without 10 min high $\mathrm{K}^{+}$stimulation. $B$, Quantification of relative GluRIII staining intensity at the NMJ with and without $10 \mathrm{~min}$ high $\mathrm{K}^{+}$depolarization. $C$, Representative images of GluRIII staining upon low-frequency ( $1 \mathrm{~Hz}$ ) or high-frequency $(10 \mathrm{~Hz})$ electrical stimulation. $\boldsymbol{D}$, Quantification of relative GluRIII intensity at synaptic terminals. $\boldsymbol{E}$, Images of GluRIIA and GluRIIIB intensity at the NMJ with and without 10 min high $\mathrm{K}^{+}$stimulation. $\boldsymbol{F}, \boldsymbol{G}$, Quantification of GluRIIA $(\boldsymbol{F})$ and GluRIIB $(\boldsymbol{G})$ staining intensities at the NMJ. ${ }^{*} p<0.05$ compared with control. All values represent the mean \pm SEM. Scale bars: $A, C, E, 5 \mu \mathrm{m}$. 
A

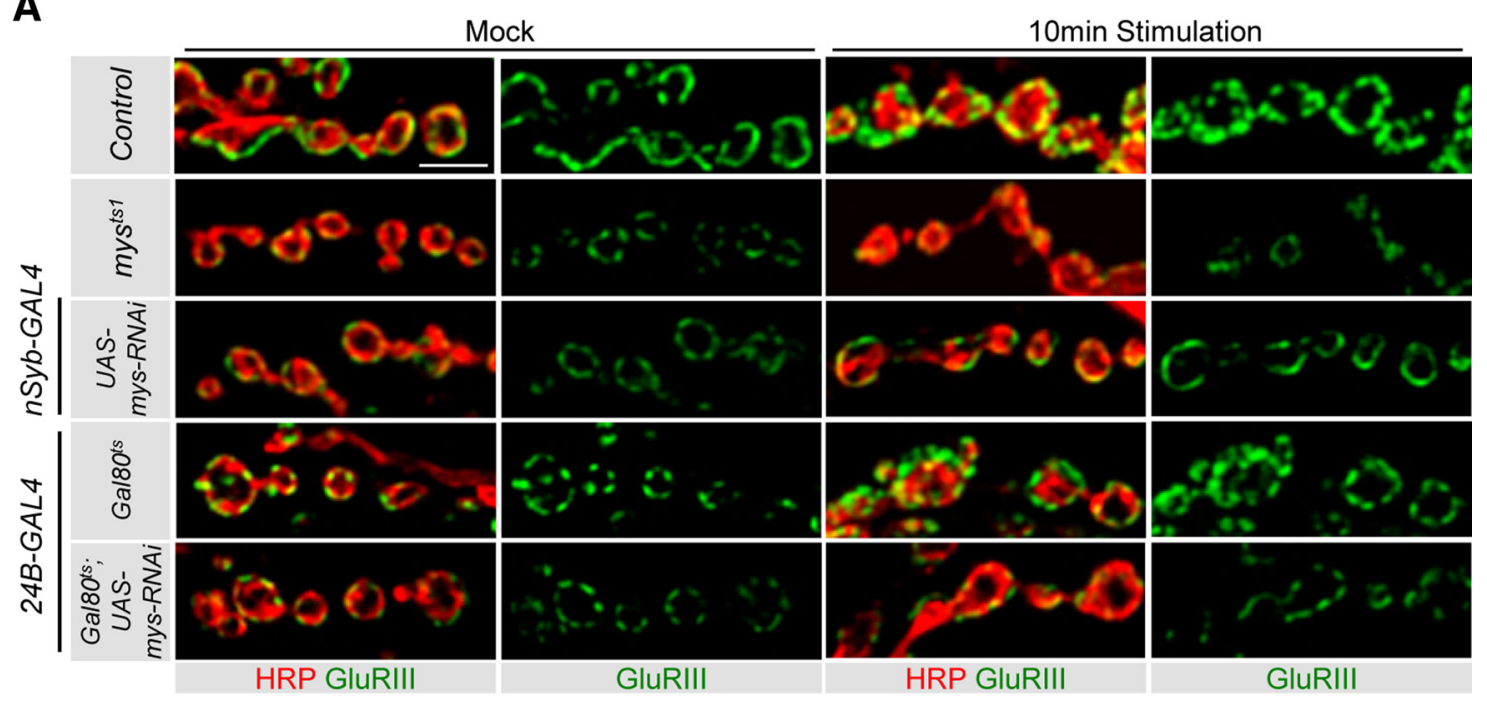

B

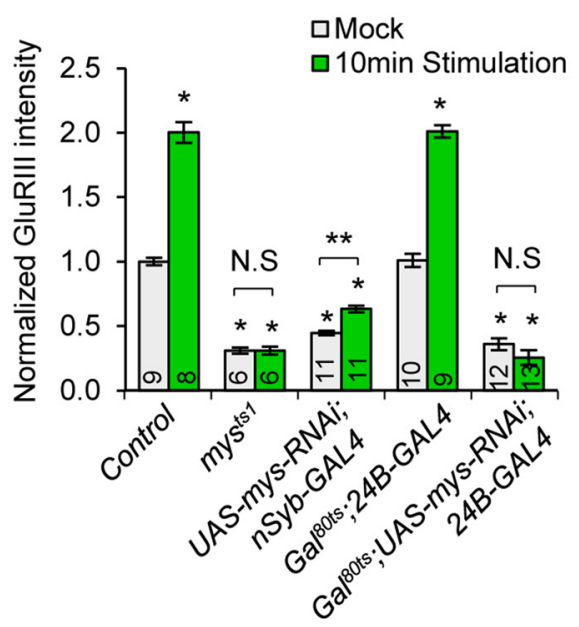

C

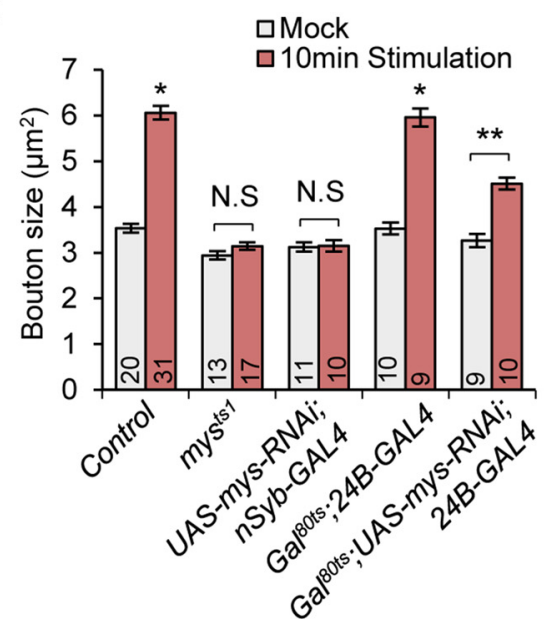

Figure 9. Presynaptic and postsynaptic $\beta$ PS integrin activation differentially affects bouton size and glutamate receptor abundance. $A$, Representative images of third-instar NMJ stained with GluRIII antibody and HRP with and without $10 \mathrm{~min} \mathrm{high} \mathrm{K}^{+}$depolarization. For genotypes containing tubulin-GAL $80^{t s}\left(G A L 80^{t s}\right)$, embryos were grown at $18^{\circ} \mathrm{C}$ until the $\mathrm{L} 1 \mathrm{stage}$ and then were shifted

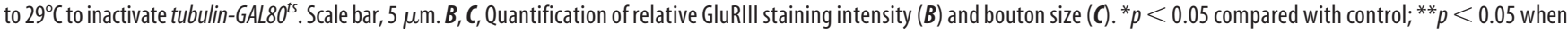
comparing the indicated conditions. All values represent the mean \pm SEM.

completely block activity-induced GluRIII elevation (Fig. 9A,B). Postsynaptic knockdown of $\beta$ PS integrin using the musclespecific 24B-GAL4 driver caused lethality. We therefore used tubulin-GAL80 ${ }^{t s}$ to inhibit $24 B-G A L 4$ expression during embryonic development and allowed $24 B-G A L 4$ to induce $m y s-R N A i$ expression after L1 stage by inactivating tubulin-GAL80 $0^{t s}$ at $29^{\circ} \mathrm{C}$. Figure 9 shows that postsynaptic knockdown of mys during the larval stage not only reduced the basal level of GluRIII but also abolished any activity-induced increase in GluRIII abundance. These results indicate that while both presynaptic and postsynaptic integrin receptors affect basal glutamate receptor levels at the synapse, activation of postsynaptic $\beta$ PS integrin receptors is absolutely required for activity-induced increase in glutamate receptor abundance. Interestingly, the opposite effect was observed for bouton size: presynaptic knockdown of mys abolished activity-induced bouton enlargement while postsynaptic knockdown of mys did not (Fig. 9A,C). Together, these data reveal that the activation of presynaptic and postsynaptic $\beta P S$ integrin receptors differentially affect bouton enlargement and glutamate receptor abundance, respectively. Our findings thus imply that bidirectional activation of $\beta$ PS integrin receptors by Shv is nec- essary to coordinate and optimize local bouton growth and maturation in response to neuronal activity.

Next, to determine whether the transcription or synthesis of new proteins contribute to this activity-dependent synaptic remodeling, we treated the NMJ with the transcriptional inhibitor actinomycin or the translational inhibitor cycloheximide. While the inhibition of transcription or protein synthesis abolished ghost bouton formation following prolonged stimulation, it did not block bouton enlargement or increase in glutamate receptor staining intensity (Fig. 10A-C). These results are consistent with findings that new bouton formation requires transcription and new protein synthesis (Fig. 10A-C; Ataman et al., 2008). Furthermore, these data suggest that synapse maturation acts via a pathway separate from the one required for new bouton formation. Because cycloheximide did not block Shv secretion following prolonged depolarization (Fig. 10D), the pathway involved in synapse maturation is likely dependent on the local release of Shv.

We asked whether the presence of Shv is sufficient to induce synapse remodeling or whether other factors released during intense synaptic activity are also required. To this end, we incu- 
A

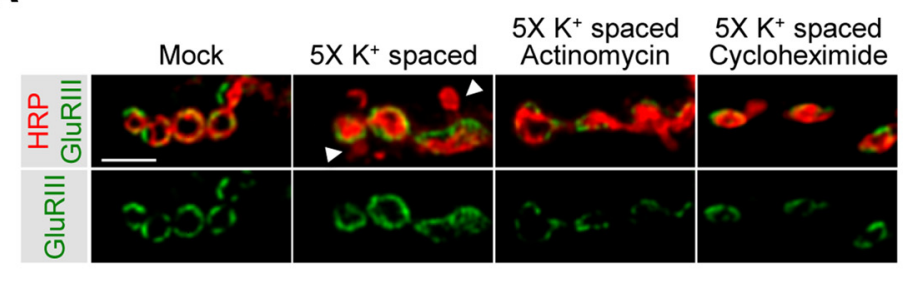

B

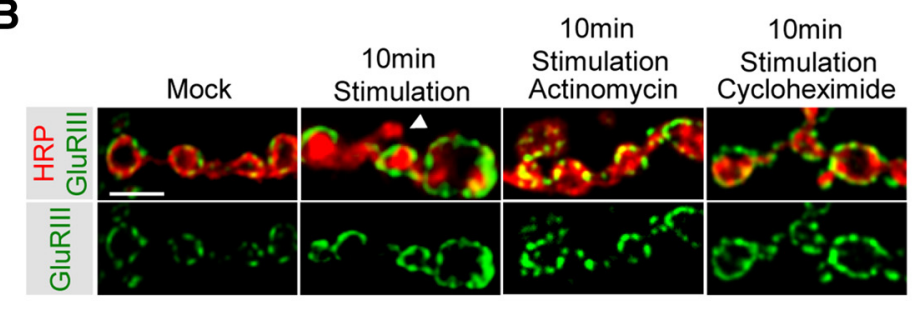

D

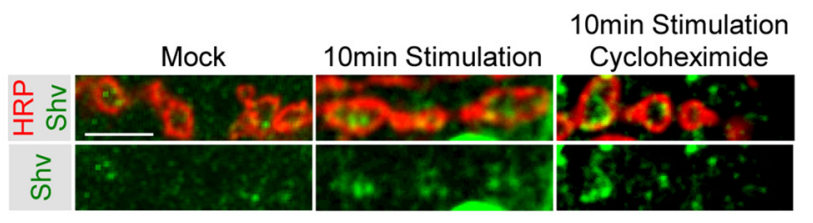

E
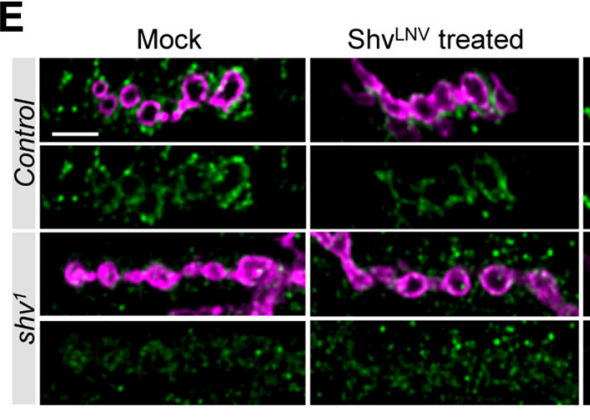

G

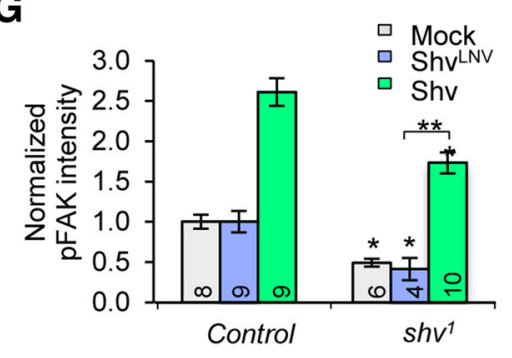

$\mathbf{J}$

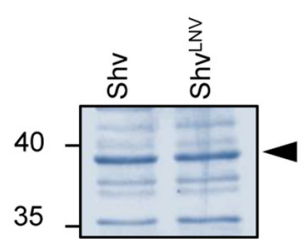

Shv treated

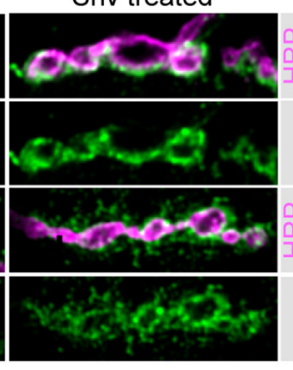

H

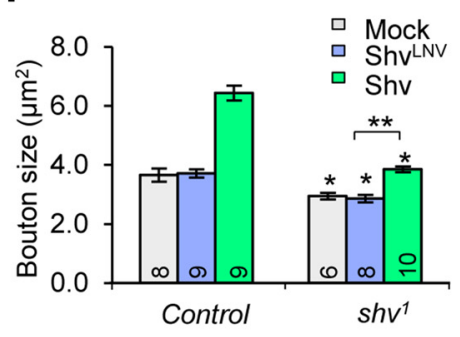

K

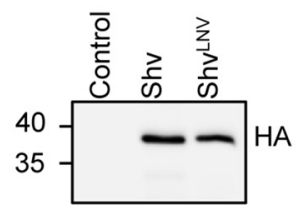

C
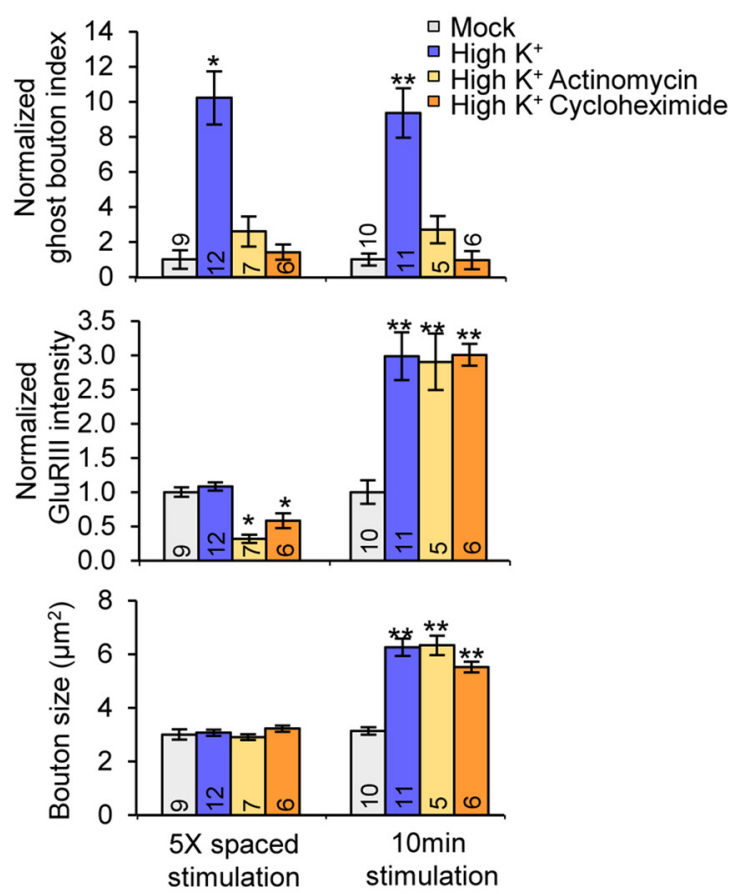

$\mathbf{F}$

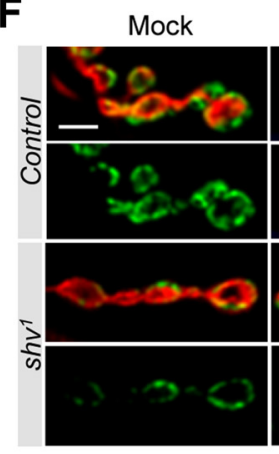

ShvLNV treated
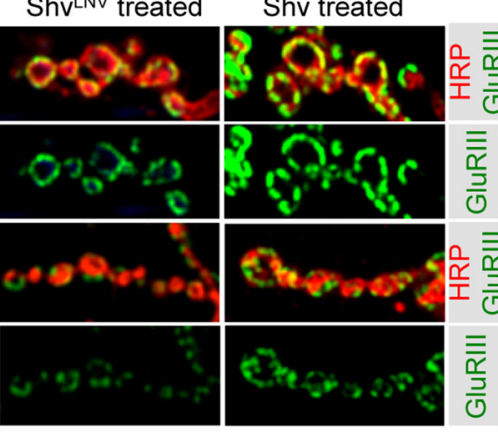

I

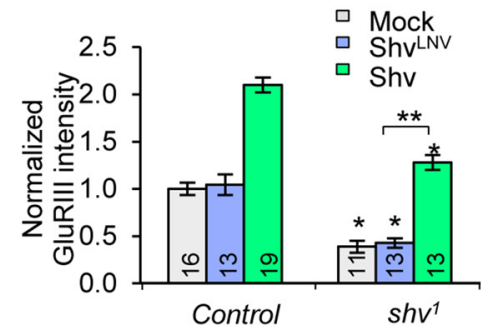

$\mathbf{L}$

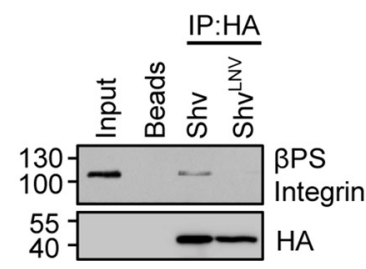

Figure 10. Extracellular incubation of Shv is sufficient to trigger synapse maturation. $A, B$, Representative images of HRP and GluRIII immunoreactivity at synaptic boutons of control NMJ subjected to $5 \times$ spaced depolarization $(\boldsymbol{A})$ and $10 \mathrm{~min}$ high $\mathrm{K}^{+}$depolarization $(\boldsymbol{B})$ in the presence of transcriptional inhibitor actinomycin or translational inhibitor cycloheximide. The ghost bouton is highlighted by a white arrowhead. C, Quantifications of normalized ghost bouton index, GluRIIIl levels, and bouton size upon $5 \times$ spaced or 10 min high $\mathrm{K}^{+}$stimulation. ${ }^{*} p<0.05$ when compared with respective mock-treated control ( $5 \times$ spaced stimulation); ${ }^{* *} p<0.05$ when compared with respective mock-treated control (10 min stimulation). $D$, Representative images of Shv release at the synapse upon prolonged high $\mathrm{K}^{+}$stimulation with and without translational inhibitor cycloheximide. Detergent-free staining condition was used. $\boldsymbol{E}$, Representative images of pFAK level changes upon incubation with purified Shv or mutated Shv (Sh ${ }^{\text {LNV }}$ ) protein. $\boldsymbol{F}$, Images representing the elevation of GluRIII level upon Shv treatment. G, Quantification of total pFAK levels at the NMJs after Shv or Shv ${ }^{\text {LNV }}$ application normalized to mock-treated control. $\boldsymbol{H}$, Enlargement of individual bouton size upon the addition of purified Shv but not (Figure legend continues.) 
A
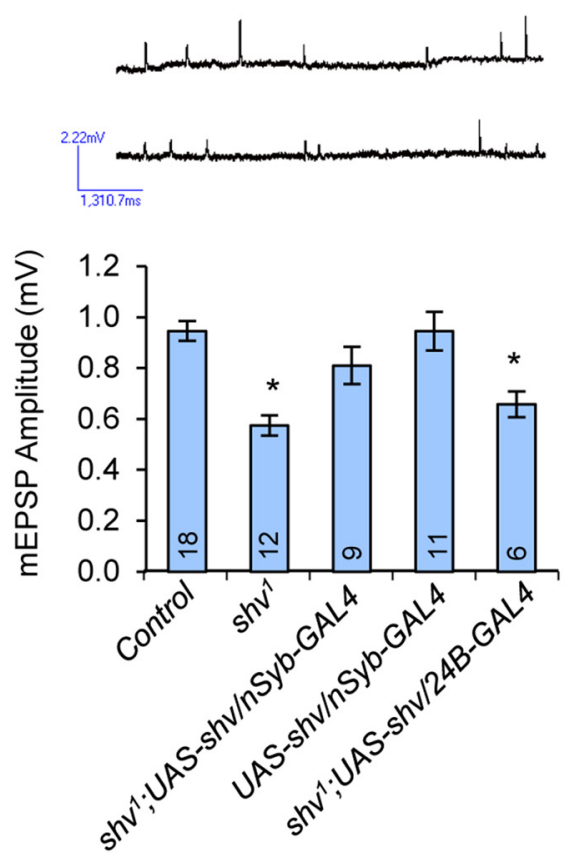

C

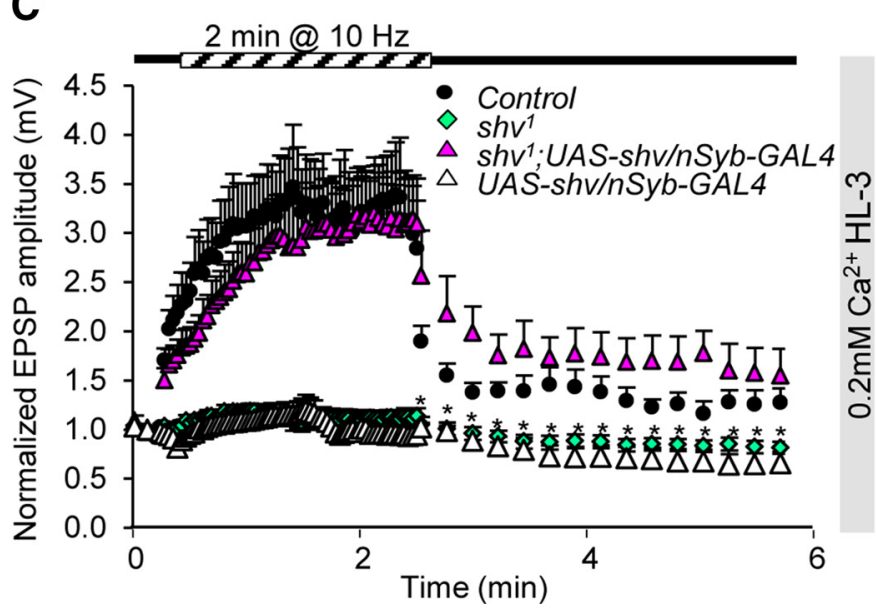

B
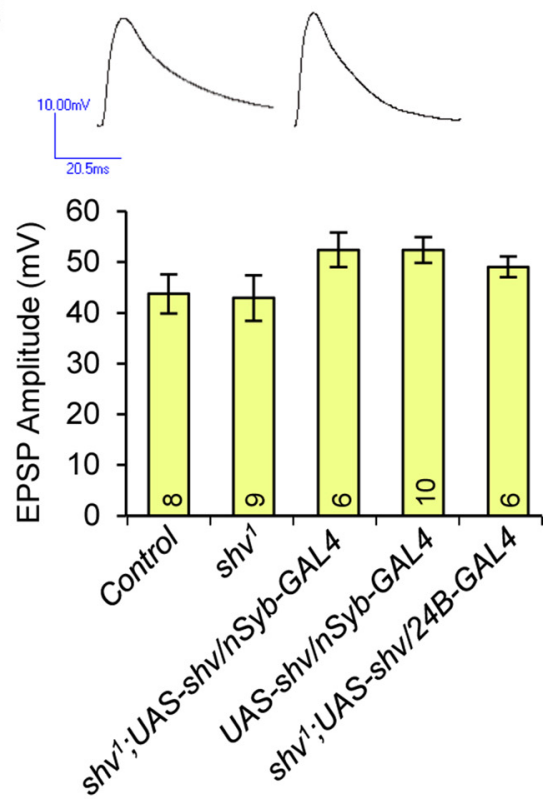

D

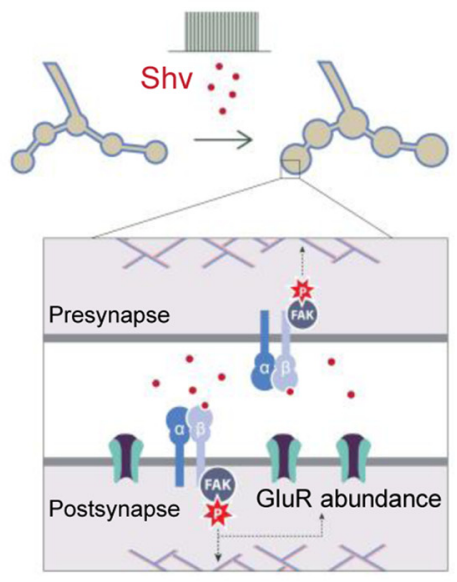

Figure 11. Shv regulates functional plasticity. $\boldsymbol{A}$, Representative mEPSP and average mEPSP amplitude recorded using an $\mathrm{HL}-3$ solution containing $0.5 \mathrm{~mm} \mathrm{Ca}^{2+} . \boldsymbol{B}$, Representative evoked EPSP recording in $\mathrm{HL} 3$ containing $0.5 \mathrm{~mm} \mathrm{Ca}{ }^{2+}$. Average EPSP was corrected using nonlinear summation. C, Normalized EPSP amplitude upon tetanus stimulation shows synaptic augmentation and post-tetanic potentiation in control NMJ, whereas shv ${ }^{7}$ failed to respond to the activity. Upregulation of Shv in shv ${ }^{7}$ mutant restored synaptic augmentation and PTP but overexpression of shv alone inhibited augmentation and PTP. Control, $n=8 ;$ shv $^{1}, n=11 ;$ shv $^{7} ;$ UAS-shv/nSyb-GAL4, $n=10 ;$ UAS-shv/nSyb-GAL4, $n=4 .{ }^{*} p<0.05$ compared with control. All values represent the mean \pm SEM. D, Model demonstrating an Shv-dependent synaptic remodeling event upon intense neuronal activity. Presynaptically released Shv activates integrin signaling pathways bidirectionally and influences local synapse growth and glutamate receptor levels at the NMJ.

bated dissected NMJ with purified Shv protein expressed in bacteria and determined the effects of extracellular Shv on synaptic morphology and maturation. In support of the hypothesis that Shv release is crucial for synapse maturation, incubating the dissected NMJ with wild-type Shv in the absence of synaptic stimulation was sufficient to elevate pFAK levels, augment the size of

\section{$\leftarrow$}

(Figure legend continued.) Shv ${ }^{\text {LNV }}$.I, Quantification of GluRIII intensity at the NMJ following Shv or Shv ${ }^{\text {LNV }}$ protein incubation normalized to mock-treated control. ${ }^{*} p<0.05$ compared with mock-treated control; ${ }^{* *} p<0.05$ when comparing the indicated conditions. All values represent the mean \pm SEM. J, Coomassie gel showing relative amounts of Shv or Shv ${ }^{\text {LNV }}$ following purification. The arrowhead points to the position of the expected protein size. $\boldsymbol{K}$, Western blot confirming the expression and isolation of Shv and Shv ${ }^{\text {LNV }}$ proteins. Western blot detected with HA antibody. L, Pull-down experiment using HA fusion proteins and fly protein lysates reveals that Shv ${ }^{\mathrm{INV}}$ does not interact with integrin. Representative blots are shown, and results were confirmed in three independent experiments. Scale bar, $5 \mu \mathrm{m}$ in all images. synaptic boutons, and boost the glutamate receptor abundance of both the control and $s h v^{I}$ mutant (Fig. 10E-I). The fact that we were able to observe these changes even in $s h v^{I}$ further implies that developmental problem was not the underlying cause of the lack of activity-dependent structural remodeling in $s h v^{1}$, but rather that the presence of extracellular Shv was necessary. In contrast, we found that incubating the NMJ with a mutant Shv protein, $S h v^{\mathrm{LNV}}$, which does not activate integrin signaling due to mutation in the putative integrin binding site (Lee et al., 2016), failed to trigger these changes (Fig. 10E-I). Note that the inability of Shv ${ }^{\mathrm{LNV}}$ to induce structural changes is not due to a difference in the amount of purified proteins (Fig. $10 \mathrm{~J}, \mathrm{~K}$ ) but rather is associated with its inability to interact with integrin, as shown in the pull-down experiment (Fig. 10L). These results further support the claim that the activation of integrin signaling by Shv is sufficient to trigger synapse remodeling. 


\section{Shv required for functional plasticity}

Having established that Shv modulates activity-induced structural modifications at the Drosophila NMJ, we asked whether Shv also alters functional plasticity. First, we measured the baseline synaptic transmission in $s h v^{I}$ mutant. Shv depletion reduced the amplitude of the miniature EPSP (mEPSP), whereas neuronal expression of Shv (but not muscle expression) rescued the phenotype of $s h v^{1}$ mutant (Fig. 11A). This decrease in mEPSP amplitude is consistent with the reduced postsynaptic glutamate receptor abundance observed with immunostaining (Fig. 8A,B). Furthermore, despite the decrease in mEPSP amplitude in $s h v^{1}$, the evoked EPSP amplitude remained normal (Fig. 11B), suggesting that homeostatic mechanism modulating synaptic response is not affected. We next examined the effects of Shv depletion on synaptic augmentation and post-tetanic potentiation (PTP), a form of synaptic plasticity. In control larvae, highfrequency stimulation at $10 \mathrm{~Hz}$ in low extracellular $\mathrm{Ca}^{2+}$ concentration normally elicits synaptic augmentation that is followed by a potentiation of the response that lasts for minutes. We found that $s h v^{I}$ showed severely reduced synaptic augmentation and impaired PTP, whereas neuronal expression of Shv in $s h v^{l}$ mutant background rescued synaptic plasticity (Fig. 11C). These results confirm that Shv is essential for functional plasticity. Interestingly, overexpression of $s h v$ alone also abolished synaptic augmentation and PTP, further implying that functional plasticity requires dynamic changes in integrin activation rather than persistent activation of integrin and downstream signaling pathways.

\section{Discussion}

In the present study, we show that Shv is a novel protein secreted at the Drosophila NMJ. We report that Shv is required during synaptic development to maintain normal synaptic growth and is released upon intense neuronal stimulation to acutely promote synapse maturation. We further demonstrate that activity-induced release of Shv by neurons activates integrin signaling bidirectionally, induces bouton enlargement, and increases postsynaptic glutamate receptor abundance (Fig. 11D). Moreover, Shv is required to achieve synaptic augmentation and maintain potentiation following tetanic stimulation. These data thus suggest that trans-synaptic Shv released in response to strong neuronal activity acts as a molecular cue that triggers activity-induced synaptic structural modifications and synapse maturation through integrin activation.

Our data reveal that presynaptically secreted Shv plays an important modulatory role in maintaining normal synaptic growth during development. We found that Shv is specifically targeted to the synapse by neurons and released at low levels in the vicinity of synaptic terminals as shown by both immunostaining with Shv antibody and Shv-GFP fusion protein puncta (Fig. 1). Furthermore, the secretion of Shv from the synapse is essential in restraining synaptic growth and promoting synapse maturation, since only the full-length Shv rescued the synaptic overgrowth and ghost bouton phenotypes of $s h v^{1}$, whereas deleting the signal peptide of Shv did not (Fig. 2). Previous studies have suggested that ghost boutons are newly formed, undifferentiated boutons in the process of maturing into functional boutons with postsynaptic glutamate receptors (Ataman et al., 2008; Menon et al., 2013). The observations that $s h v^{1}$ mutants have more synaptic boutons and ghost boutons suggest that presynaptically released Shv is not required for new bouton formation but rather restricts synaptic growth during development.

The low amounts of Shv present extracellularly likely activate $\beta$ PS integrin signaling both presynaptically and postsynaptically to modulate synaptic growth. This is supported by our previous work showing that activation of $\beta$ PS integrin receptors by Shv in
S2 cells (Lee et al., 2016), data that Shv can biochemically and genetically interact with $\beta$ PS integrin to regulate synaptic growth, and that presynaptic knockdown of Shv significantly reduced pFAK levels in both presynaptic terminals and postsynaptic muscle (Fig. 3). We thus envision that Shv, once released at presynaptic terminals, is a trans-synaptic molecule that acts on $\beta$ PS integrin receptors bidirectionally to affect synaptic growth through signaling pathways downstream of $\beta$ PS integrin receptors. As there are multiple integrin ligands shown to affect synaptic growth and different integrin receptors at the NMJ (Broadie et al., 2011), it is likely that Shv acts in concert with other integrin ligands to coordinate and optimize integrin activation and synaptic growth during development.

Although the involvement of integrin in synaptic growth and plasticity has been studied extensively (Beumer et al., 1999; Rohrbough et al., 2000; Chavis and Westbrook, 2001; Beumer et al., 2002; Kramár et al., 2006; Tsai et al., 2008, 2012a,b), the identity of an extracellular ligand that modulates integrin signaling in response to synaptic activity remains to be elucidated. Excitingly, we found that persistent synaptic activity or highfrequency stimulation induced the release of Shv, which led to a robust increase in pFAK level and synaptic bouton enlargement, a process normally associated with synaptic maturation. Furthermore, the incubation of purified Shv protein in dissected larval NMJ elevated the levels of pFAK and enlarged the size of synaptic boutons, while the mutant Shv ${ }^{\mathrm{LNV}}$ that cannot interact with $\beta \mathrm{PS}$ failed to elicit such changes (Fig. 10E-I). These results suggest that the extracellular presence of Shv is an instructive cue that is sufficient to acutely activate integrin signaling and induce synaptic structural modification. Moreover, our finding that the absence of Shv failed to elicit multiple phases of activity-induced synaptic plasticity, including initial augmentation during tetanus and PTP, supports the claim that Shv is required for synaptic plasticity.

To date, aside from Shv, laminin A is the only known integrin ligand with its release regulated in an activity-dependent manner at the Drosophila NMJ. However, laminin release by postsynaptic muscles is instead downregulated by synaptic activity and crawling, resulting in reduced pFAK through its retrograde action on presynaptically expressed $\beta_{\nu}$ integrin receptors (Tsai et al., 2012a). Here we find that the release of Shv is stimulated by strong synaptic activity and acts independently of $\beta_{\nu}$ integrin receptors (Fig. 6). Interestingly, we also found that the spaced potassium depolarization paradigm, which has been shown to induce new bouton formation and synaptic growth through the release of wingless (Wg; Ataman et al., 2008), did not trigger Shv release or elevate pFAK at the NMJ (Fig. 5). Furthermore, we found that while new bouton formation was blocked by transcription or translational inhibitors similar to those in previous reports (Fig. 10A-C; Ataman et al., 2008), bouton maturation in the form of bouton enlargement and increase in glutamate receptor abundance was not affected (Fig. 10C). Together, these data support a model in which neurons activate different programs to differentially modulate synaptic growth and maturation in response to different synaptic demands.

We propose that mild or patterned neuronal activity triggers release of factors such as $\mathrm{Wg}$ and downregulates laminin release from muscles to initially promote synaptic growth and allow synaptic expansion (increase the number of boutons); persistent or intense synaptic activity then leads to the release of Shv from neurons to activate $\beta$ PS integrin signaling bidirectionally to promote local synapse maturation. By selectively reducing presynaptic or postsynaptic $\beta$ PS integrin receptors (Fig. 9), we demonstrate that presynaptic $\beta$ PS signaling is absolutely required for bouton enlargement following 
neuronal activity, whereas postsynaptic $\beta$ PS activation is required for the activity-induced increase in glutamate receptor abundance. Activation of $\beta$ PS integrin signaling may lead to local synaptic enlargement in bouton size by promoting actin branching (Levi et al., 2006; Legate et al., 2009), which has been associated with growth in the size of boutons (Collins and DiAntonio, 2004). The exact mechanism for how activation of $\beta$ PS integrin leads to an increase in glutamate receptor abundance at the Drosophila NMJ is not clear, but mutations that reduce the levels of $\beta$ PS integrin have also been shown to diminish glutamate receptor levels (Liebl and Featherstone, 2005). Secreted extracellular protein such as mind-the-gap, which has been suggested to recruit integrin receptor to the synaptic cleft, also affects synaptic localization of glutamate receptor clusters (Rohrbough et al., 2007; Rushton et al., 2009). Furthermore, PS integrin receptors have been reported to associate with Discs large, Fasciclin II cell adhesion molecule, and CaMKII, which are known to affect synaptic glutamate receptor assembly and anchoring (Beumer et al., 1999; Albin and Davis, 2004; Chen and Featherstone, 2005; Chen et al., 2005). Understanding the downstream mechanism by which Shv-dependent integrin activation modulates the localization of glutamate receptor to promote the stability of synaptic structure during synaptic remodeling remains an interesting area to study in the future. It will also be particularly interesting to elucidate how neuronal activity triggers $S h v$ release. As a robust neuronal circuitry depends on the ability of neurons to dynamically adjust synaptic strength and modify synaptic structure in response to synaptic demand, elucidating how Shv functions in activity-induced synaptic plasticity has implications for understanding mechanisms underlying cognition and psychiatric disorders.

\section{References}

Albin SD, Davis GW (2004) Coordinating structural and functional synapse development: postsynaptic p21-activated kinase independently specifies glutamate receptor abundance and postsynaptic morphology. J Neurosci 24:6871-6879. CrossRef Medline

Arikkath J (2010) N-cadherin: stabilizing synapses. J Cell Biol 189:397-398. CrossRef Medline

Ataman B, Ashley J, Gorczyca M, Ramachandran P, Fouquet W, Sigrist SJ, Budnik V (2008) Rapid activity-dependent modifications in synaptic structure and function require bidirectional Wnt signaling. Neuron 57: 705-718. CrossRef Medline

Bahr BA, Staubli U, Xiao P, Chun D, Ji ZX, Esteban ET, Lynch G (1997) Arg-Gly-Asp-Ser-selective adhesion and the stabilization of long-term potentiation: pharmacological studies and the characterization of a candidate matrix receptor. J Neurosci 17:1320-1329. Medline

Berninger B, Bi GQ (2002) Synaptic modification in neural circuits: a timely action. Bioessays 24:212-222. CrossRef Medline

Beumer KJ, Rohrbough J, Prokop A, Broadie K (1999) A role for PS integrins in morphological growth and synaptic function at the postembryonic neuromuscular junction of Drosophila. Development 126:5833-5846. Medline

Beumer K, Matthies HJ, Bradshaw A, Broadie K (2002) Integrins regulate DLG/FAS2 via a CaM kinase II-dependent pathway to mediate synapse elaboration and stabilization during postembryonic development. Development 129:3381-3391. Medline

Bourne JN, Harris KM (2008) Balancing structure and function at hippocampal dendritic spines. Annu Rev Neurosci 31:47-67. CrossRef Medline

Broadie K, Baumgartner S, Prokop A (2011) Extracellular matrix and its receptors in Drosophila neural development. Dev Neurobiol 71:11021130. CrossRef Medline

Brown NH (1993) Integrins hold Drosophila together. Bioessays 15:383390. CrossRef Medline

Campbell ID, Humphries MJ (2011) Integrin structure, activation, and interactions. Cold Spring Harb Perspect Biol 3:a004994. CrossRef Medline

Chan CS, Weeber EJ, Kurup S, Sweatt JD, Davis RL (2003) Integrin requirement for hippocampal synaptic plasticity and spatial memory. J Neurosci 23:7107-7116. Medline
Chan CS, Weeber EJ, Zong L, Fuchs E, Sweatt JD, Davis RL (2006) Beta 1-integrins are required for hippocampal AMPA receptor-dependent synaptic transmission, synaptic plasticity, and working memory. J Neurosci 26:223-232. CrossRef Medline

Chavis P, Westbrook G (2001) Integrins mediate functional presynaptic and postsynaptic maturation at a hippocampal synapse. Nature 411:317321. CrossRef Medline

Chen CK, Bregere C, Paluch J, Lu JF, Dickman DK, Chang KT (2014) Activity-dependent facilitation of Synaptojanin and synaptic vesicle recycling by the Minibrain kinase. Nat Commun 5:4246. CrossRef Medline

Chen K, Featherstone DE (2005) Discs-large (DLG) is clustered by presynaptic innervation and regulates postsynaptic glutamate receptor subunit composition in Drosophila. BMC Biol 3:1. CrossRef Medline

Chen K, Merino C, Sigrist SJ, Featherstone DE (2005) The 4.1 protein coracle mediates subunit-selective anchoring of Drosophila glutamate receptors to the postsynaptic actin cytoskeleton. J Neurosci 25:6667-6675. CrossRef Medline

Chklovskii DB, Mel BW, Svoboda K (2004) Cortical rewiring and information storage. Nature 431:782-788. CrossRef Medline

Chun D, Gall CM, Bi X, Lynch G (2001) Evidence that integrins contribute to multiple stages in the consolidation of long term potentiation in rat hippocampus. Neuroscience 105:815-829. CrossRef Medline

Cohen S, Greenberg ME (2008) Communication between the synapse and the nucleus in neuronal development, plasticity, and disease. Annu Rev Cell Dev Biol 24:183-209. CrossRef Medline

Collins CA, DiAntonio A (2004) Coordinating synaptic growth without being a nervous wreck. Neuron 41:489-491. CrossRef Medline

Dani N, Zhu H, Broadie K (2014) Two protein N-acetylgalactosaminyl transferases regulate synaptic plasticity by activity-dependent regulation of integrin signaling. J Neurosci 34:13047-13065. CrossRef Medline

DiAntonio A, Petersen SA, Heckmann M, Goodman CS (1999) Glutamate receptor expression regulates quantal size and quantal content at the Drosophila neuromuscular junction. J Neurosci 19:3023-3032. Medline

Dityatev A, Schachner M (2003) Extracellular matrix molecules and synaptic plasticity. Nat Rev Neurosci 4:456-468. CrossRef Medline

Dityatev A, Schachner M, Sonderegger P (2010) The dual role of the extracellular matrix in synaptic plasticity and homeostasis. Nat Rev Neurosci 11:735-746. CrossRef Medline

Fiala JC, Spacek J, Harris KM (2002) Dendritic spine pathology: cause or consequence of neurological disorders? Brain Res Brain Res Rev 39:29_ 54. CrossRef Medline

Fogerty FJ, Fessler LI, Bunch TA, Yaron Y, Parker CG, Nelson RE, Brower DL, Gullberg D, Fessler JH (1994) Tiggrin, a novel Drosophila extracellular matrix protein that functions as a ligand for Drosophila alpha PS2 beta PS integrins. Development 120:1747-1758. Medline

Frank CA, Kennedy MJ, Goold CP, Marek KW, Davis GW (2006) Mechanisms underlying the rapid induction and sustained expression of synaptic homeostasis. Neuron 52:663-677. CrossRef Medline

Gotwals PJ, Paine-Saunders SE, Stark KA, Hynes RO (1994) Drosophila integrins and their ligands. Curr Opin Cell Biol 6:734-739. CrossRef Medline

Graner MW, Bunch TA, Baumgartner S, Kerschen A, Brower DL (1998) Splice variants of the Drosophila PS2 integrins differentially interact with RGD-containing fragments of the extracellular proteins tiggrin, ten-m, and D-laminin 2. J Biol Chem 273:18235-18241. CrossRef Medline

Grooms SY, Jones LS (1997) RGDS tetrapeptide and hippocampal in vitro kindling in rats: evidence for integrin-mediated physiological stability. Neurosci Lett 231:139-142. CrossRef Medline

Harburger DS, Calderwood DA (2009) Integrin signalling at a glance. J Cell Sci 122:159-163. CrossRef Medline

Holtmaat A, Svoboda K (2009) Experience-dependent structural synaptic plasticity in the mammalian brain. Nat Rev Neurosci 10:647-658. CrossRef Medline

Huang Z, Shimazu K, Woo NH, Zang K, Müller U, Lu B, Reichardt LF (2006) Distinct roles of the beta 1-class integrins at the developing and the mature hippocampal excitatory synapse. J Neurosci 26:11208-11219. CrossRef Medline

Hunt LC, Demontis F (2013) Whole-mount immunostaining of Drosophila skeletal muscle. Nat Protoc 8:2496-2501. CrossRef Medline

Inoue Y, Hayashi S (2007) Tissue-specific laminin expression facilitates integrin-dependent association of the embryonic wing disc with the trachea in Drosophila. Dev Biol 304:90-101. CrossRef Medline 
Je HS, Yang F, Zhou J, Lu B (2006) Neurotrophin 3 induces structural and functional modification of synapses through distinct molecular mechanisms. J Cell Biol 175:1029-1042. CrossRef Medline

Kim K, Lakhanpal G, Lu HE, Khan M, Suzuki A, Hayashi MK, Narayanan R, Luyben TT, Matsuda T, Nagai T, Blanpied TA, Hayashi Y, Okamoto K (2015) A temporary gating of actin remodeling during synaptic plasticity consists of the interplay between the kinase and structural functions of CaMKII. Neuron 87:813-826. CrossRef Medline

Koon AC, Ashley J, Barria R, DasGupta S, Brain R, Waddell S, Alkema MJ, Budnik V (2011) Autoregulatory and paracrine control of synaptic and behavioral plasticity by octopaminergic signaling. Nat Neurosci 14:190199. CrossRef Medline

Kramár EA, Lin B, Rex CS, Gall CM, Lynch G (2006) Integrin-driven actin polymerization consolidates long-term potentiation. Proc Natl Acad Sci U S A 103:5579-5584. CrossRef Medline

Lamprecht R, LeDoux J (2004) Structural plasticity and memory. Nat Rev Neurosci 5:45-54. CrossRef Medline

Lee JY, Chen JY, Shaw JL, Chang KT (2016) Maintenance of stem cell niche integrity by a novel activator of integrin signaling. PLoS Genet 12: e1006043. CrossRef Medline

Legate KR, Wickström SA, Fässler R (2009) Genetic and cell biological analysis of integrin outside-in signaling. Genes Dev 23:397-418. CrossRef Medline

Levi BP, Ghabrial AS, Krasnow MA (2006) Drosophila talin and integrin genes are required for maintenance of tracheal terminal branches and luminal organization. Development 133:2383-2393. CrossRef Medline

Liebl FL, Featherstone DE (2005) Genes involved in Drosophila glutamate receptor expression and localization. BMC Neurosci 6:44. CrossRef Medline

McGeachie AB, Cingolani LA, Goda Y (2011) Stabilising influence: integrins in regulation of synaptic plasticity. Neurosci Res 70:24-29. CrossRef Medline

Menon KP, Carrillo RA, Zinn K (2013) Development and plasticity of the Drosophila larval neuromuscular junction. Wiley Interdiscip Rev Dev Biol 2:647-670. CrossRef Medline

Mitra SK, Hanson DA, Schlaepfer DD (2005) Focal adhesion kinase: in command and control of cell motility. Nat Rev Mol Cell Biol 6:56-68. CrossRef Medline

Mosca TJ (2015) On the Teneurin track: a new synaptic organization molecule emerges. Front Cell Neurosci 9:204. CrossRef Medline

Okumura T, Takeda K, Taniguchi K, Adachi-Yamada T (2014) betanu integrin inhibits chronic and high level activation of JNK to repress senescence phenotypes in Drosophila adult midgut. PLoS One 9:e89387. CrossRef Medline

Pauli A, Althoff F, Oliveira RA, Heidmann S, Schuldiner O, Lehner CF, Dickson BJ, Nasmyth K (2008) Cell-type-specific TEV protease cleavage reveals cohesin functions in Drosophila neurons. Dev Cell 14:239-251. CrossRef Medline
Petersen SA, Fetter RD, Noordermeer JN, Goodman CS, DiAntonio A (1997) Genetic analysis of glutamate receptors in Drosophila reveals a retrograde signal regulating presynaptic transmitter release. Neuron 19: 1237-1248. CrossRef Medline

Rohrbough J, Grotewiel MS, Davis RL, Broadie K (2000) Integrin-mediated regulation of synaptic morphology, transmission, and plasticity. J Neurosci 20:6868-6878. Medline

Rohrbough J, Rushton E, Woodruff E 3rd, Fergestad T, Vigneswaran K, Broadie K (2007) Presynaptic establishment of the synaptic cleft extracellular matrix is required for post-synaptic differentiation. Genes Dev 21:2607-2628. CrossRef Medline

Rushton E, Rohrbough J, Broadie K (2009) Presynaptic secretion of mindthe-gap organizes the synaptic extracellular matrix-integrin interface and postsynaptic environments. Dev Dyn 238:554-571. CrossRef Medline

Stäubli U, Chun D, Lynch G (1998) Time-dependent reversal of longterm potentiation by an integrin antagonist. J Neurosci 18:3460-3469. Medline

Trachtenberg JT, Chen BE, Knott GW, Feng G, Sanes JR, Welker E, Svoboda $\mathrm{K}$ (2002) Long-term in vivo imaging of experience-dependent synaptic plasticity in adult cortex. Nature 420:788-794. CrossRef Medline

Tsai PI, Kao HH, Grabbe C, Lee YT, Ghose A, Lai TT, Peng KP, Van Vactor D, Palmer RH, Chen RH, Yeh SR, Chien CT (2008) Fak56 functions downstream of integrin alphaPS3betanu and suppresses MAPK activation in neuromuscular junction growth. Neural Dev 3:26. CrossRef Medline

Tsai PI, Wang M, Kao HH, Cheng YJ, Lin YJ, Chen RH, Chien CT (2012a) Activity-dependent retrograde laminin A signaling regulates synapse growth at Drosophila neuromuscular junctions. Proc Natl Acad Sci U S A 109:17699-17704. CrossRef Medline

Tsai PI, Wang M, Kao HH, Cheng YJ, Walker JA, Chen RH, Chien CT (2012b) Neurofibromin mediates FAK signaling in confining synapse growth at Drosophila neuromuscular junctions. J Neurosci 32:1697116981. CrossRef Medline

Vasin A, Zueva L, Torrez C, Volfson D, Littleton JT, Bykhovskaia M (2014) Synapsin regulates activity-dependent outgrowth of synaptic boutons at the Drosophila neuromuscular junction. J Neurosci 34:10554-10563. CrossRef Medline

Wagh DA, Rasse TM, Asan E, Hofbauer A, Schwenkert I, Dürrbeck H, Buchner S, Dabauvalle MC, Schmidt M, Qin G, Wichmann C, Kittel R, Sigrist SJ, Buchner E (2006) Bruchpilot, a protein with homology to ELKS/ CAST, is required for structural integrity and function of synaptic active zones in Drosophila. Neuron 49:833-844. CrossRef Medline

Xie Z, Srivastava DP, Photowala H, Kai L, Cahill ME, Woolfrey KM, Shum CY, Surmeier DJ, Penzes P (2007) Kalirin-7 controls activity-dependent structural and functional plasticity of dendritic spines. Neuron 56:640 656. CrossRef Medline

Yamagata M, Sanes JR, Weiner JA (2003) Synaptic adhesion molecules. Curr Opin Cell Biol 15:621-632. CrossRef Medline 